\title{
DEFORMATION OF SUBMANIFOLDS OF HOMOGENEOUS SPACES
}

\author{
GARY R. JENSEN
}

\section{Introduction}

In his paper [6], P. Griffiths considered what he called $k$ th order contact between two $p$-dimensional submanifolds $f, \bar{f}: S \rightarrow M$ of a homogeneous space $M=G / G_{0}$, (cf. p. 799). He considered the question of how to determine when two such submanifolds have $k$ th order contact, and also whether it is true that, for large enough $k, k$ th order contact implies congruence.

As a method of attack on these problems Griffiths discussed E. Cartan's method of moving frames, by which he meant finding appropriate "lifts" of $f$ and $\bar{f}$ to $G$ and then using the pull-back of the Maurer-Cartan form of $G$ to obtain local invariants which would provide necessary and sufficient criteria for when two submanifolds have $k$ th order contact. Although he successfully carried out this approach for several examples, a general method, and especially an appropriate method for lifting $f$ to $G$, remained an open question.

In my monograph [7], I considered these problems raised by Griffiths, but for a different notion of contact. In his 1938 review of Cartan's book [3], $\mathbf{H}$. Weyl pointed out that there are two natural notions of contact in a homogeneous space. He called them "fixed parameter contact", which is the version used by Griffiths; and "unfixed parameter contact", which is the version that I used. For unfixed parameter contact, the problems of $k$ th order contact and congruence were solved in [7] using the method of higher order frames. These frames consist of a nested sequence of submanifolds of $G$, cross sections of which constitute the appropriate "lifts" sought by Griffiths. The methods are quite general and can be carried out whenever (but not only then) the isotropy subgroup of $G$ is compact.

In classical differential geometry of surfaces in Euclidean space, the principal curvatures are invariants of the unfixed parameter version of contact,

Communicated by P. A. Griffiths, March 29, 1980. 
while the first and second fundamental forms are invariants of the fixed parameter version of contact. Any two surfaces have first order unfixed parameter rigid motion contact, but they have first order fixed parameter rigid motion contact if and only if they are isometric. A relationship between the two versions of contact emerges when it is recognized that from the two principal curvatures can be constructed the Gaussian curvature, which is an invariant of the fixed parameter contact.

The purpose of this paper is to elucidate the exact relationship between the two versions of contact, and to solve the problems raised by Griffiths for the fixed parameter case by making use of the higher order frame construction developed for the unfixed parameter case.

In the first three sections the two versions of contact are defined and the exact relationship between them is described, (Proposition 2). Following Cartan I have called the unfixed parameter contact " $G$-contact", (the notion of [3]), and the fixed parameter contact I have called " $G$-deformation", (the notion of [1] and [2]).

$\$ 4$ contains the general method for attacking the $G$-deformation problem. This attack consists of deriving a system of exterior differential equations on the parameter space $S$ of $f, \bar{f}: S \rightarrow M$, relative to a pair of related lifts $u, \bar{u}$ : $S \rightarrow G$ of $f$ and $\bar{f}$, respectively. This system of equations is such that $f$ and $\bar{f}$ are $G$-deformations of a given order if and only if his system of equations is satisfied.

In $\$ 5$ it is shown how to view this system as an exterior differential system on $G \times G$ or $L_{k} \times G$, where $L_{k} \subseteq G$ is the space of $k$ th order frames on $f$. The Cartan-Kähler Theory can then be used to study these equations. The section concludes with a proof of the proposition that, under quite general conditions, some finite order of $G$-deformation implies congruence, (even for $C^{\infty}$ submanifolds).

In the second part of the paper the general method is applied and worked out in detail for the case of surfaces in $\mathbf{R}^{3}$ under the action of the equiaffine group, $S L(3 ; \mathbf{R}) \mathbf{R}^{3}$. This example was chosen because surfaces in $\mathbf{R}^{3}$ are familiar objects, the higher order frame construction has been completely carried out for them in [7], and the isotropy subgroup $S L(3 ; \mathbf{R})$ is noncompact. This last feature is important because whenever the isotropy $G_{0}$ is compact there exists a $G$-invariant Riemannian metric on $M$, and then the first order $G$-deformation problem is just the isometric imbedding problem. When $G_{0}$ is noncompact, there are no familiar fundamental forms around providing invariants for the deformation problem. One must turn to the $G$-contact frames and invariants. 
It is a pleasure to thank Phillip Griffiths for his suggestions for revising this paper, in particular his suggestion to expand $\$ \S 4$ and 5 to a description of the general case rather than just for the equiaffine space example. I recommend some recent papers by C. Schiemangk, R. Sulanke and A. Švec on the higher order frame construction, [9], [10], [11]. Finally, I must acknowledge a great debt to several papers and books of Elie Cartan. In so far as I understand what he did, the methods described here are the methods he used in his study [1] of the projective deformation of surfaces.

\section{GENERAL THEORY OF DEFORMATIONS}

\section{Notation}

In this paper we assume all maps and manifolds to be differentiable of Class $C^{\infty}$, unless other assumptions are made explicitly. In most cases class $C^{r}$ for some finite $r$ would be adequate, but in the present context there seems to be no point in stressing the weakest differentiability assumptions needed. Whenever we apply the Cartan-Kähler theorem we must, of course, assume everything to be real analytic. Such assumptions will be stated whenever they are made.

Vectors in $\mathbf{R}^{m}$ are denoted with $m \times 1$ matrices. For any positive integer $p<m$, the Grassmann manifold $G_{m p}$ of unoriented $p$-dimensional linear subspaces of $\mathbf{R}^{m}$ can be represented as the orbit space $\tilde{\mathbf{R}}^{m \times p} / G L(p ; \mathbf{R})$, where $\tilde{\mathbf{R}}^{m \times p}$ denotes the space of all $m \times p$ real matrices of rank equal to $p$. The general linear group $G L(p ; \mathbf{R})$ acts on $\tilde{\mathbf{R}}^{m \times p}$ from the right by ordinary matrix multiplication. For any matrix $X \in \tilde{\mathbf{R}}^{m \times p}$, we let $[X]$ denote the equivalence of class $X$ under the action of $G L(p ; \mathbf{R})$. If $X, Y \in \tilde{\mathbf{R}}^{m \times p}$, then $[X]=[Y]$ iff $X=Y A$ for some $A \in G L(p ; \mathbf{R})$.

$G L(m ; \mathbf{R})$ acts as a Lie transformation group on $G_{m, p}$ by left matrix multiplication. If $A \in G L(m ; \mathbf{R})$, and $[X] \in G_{m, p}$, then $A \cdot[X]=[A X]$.

The following local coordinate chart in $G_{m, p}$ will be referred to as the standard local coordinate system in $G_{m, p}$. Let $U_{0}=\left\{[X] \in G_{m p}\right.$ : the first $p$ rows of $X$ are linearly independent $\}$. For any $X \in \tilde{\mathbf{R}}^{m \times p}$, write $X=\left(\begin{array}{c}A \\ Y\end{array}\right)$, where $A \in \mathbf{R}^{p \times p}$ and $Y \in \mathbf{R}^{(m-p) \times p}$. Then $U_{0}=\left\{\left[\left[_{Y}^{A}\right] \in G_{m, p}: A \in\right.\right.$ $G L(p ; \mathbf{R})\}=\left\{\left[{ }_{Y}^{I}\right]: I=p \times p\right.$ identity, $\left.Y \in \mathbf{R}^{(m-p) \times p}\right\}$. The map $y_{0}: U_{0} \rightarrow$ $\mathbf{R}^{(m-p) \times p}$ given by $y_{0}\left(\left[{ }_{Y}^{A}\right]\right)=Y A^{-1}$ is a homeomorphism, and $\left(U_{0}, y_{0}\right)$ is the standard local coordinate system of $G_{m p}$.

For bundle notation and terminology we shall follow [8]. If $M$ is an $m$-dimensional manifold, then $L(M)$ denotes the $G L(m ; \mathbf{R})$-principal bundle of linear frames on $M$. We let $G_{m, p}(M)$ denote the fiber bundle of all 
$p$-dimensional subspaces of the tangent space of $M$ at any point of $M$; in short, the bundle of all $p$-dimensional tangent planes to $M$.

The bundle $G_{m, p}(M)$ is associated to $L(M)$ with the standard fiber $G_{m p}$ on which the structure group $G L(m, \mathbf{R})$ acts as described above. Thus $G_{m, p}(M)$ $=L(M) x_{G L(m ; R)} G_{m p}$. A tangent $p$-plane $P$ at the point $x$ in $M$ is denoted by $[u,[X]] \in L(M) x_{G L(m ; R)} G_{m, p}$. Geometrically, $u=\left(e_{1}, \cdots, e_{m}\right)$ is a basis of $M_{x}$, and $X=\left(x_{\alpha}^{i}\right), 1 \leqslant i \leqslant m, 1 \leqslant \alpha \leqslant p$, is an $m \times p$ matrix such that $P$ is spanned by the $p$ vectors $v_{\alpha}=\Sigma_{i} x_{\alpha}^{i} e_{i} \in M_{x}$.

\section{Contact}

Let $f: S \rightarrow M$ be a $p$-dimensional submanifold of an $m$-dimensional manifold $M$, where $1 \leqslant p \leqslant m$. Then $f$ induces an imbedding $T_{f}: S \rightarrow G_{m, p}(M)$ given by $T_{f}(s)=f_{*} S_{s}, s \in S$.

We define the higher order Grassmann bundles on $M$ inductively by $M^{0}=M, M^{r+1}=G_{m_{r}, p}\left(M^{R}\right)$ for any integer $r \geqslant 0$, where $m_{r}=\operatorname{dim} M^{r}$. A sequence of induced maps $T_{f}^{r}: S \rightarrow M^{r}$ is defined inductively by $T_{f}^{o}=f$, $T_{f}^{r+1}=T_{T_{f}^{r}}$, for any integer $r \geqslant 0$.

Let $\bar{f}: \bar{S} \rightarrow M$ be another $p$-diménsional submanifold of $M$, and $r$ a positive integer.

Definition. (Compare with [3, §16, pp. 16-17].) $f$ and $\bar{f}$ have contact of at least order $r$ at $s \in S$ and $\bar{s} \in \bar{S}$ if $T_{f}^{r}(s)=T_{\bar{f}}^{r}(\bar{s})$.

In order to get a more detailed view of the meaning of contact, we shall see what contact means in terms of appropriately chosen local coordinates. We shall use the following index conventions: $1 \leqslant i, j, k<m ; 1 \leqslant \alpha, \beta \leqslant p$; $p+1 \leqslant a, b, c \leqslant m$.

Fix a point $o \in S$, and let $y=\left(y^{1}, \cdots, y^{m}\right)$ be a local coordinate system in $M$ centered at $f(o)$. If we let $f^{i}=y^{i} \circ f$, then as $f$ is an imbedding, we may assume (reindexing the $y^{i}$ if necessary) that $d f^{1} \wedge \cdots \wedge d f^{p}(o) \neq 0$. Then $x=\left(f^{1}, \cdots, f^{p}\right)$ defines a local coordinate system in $S$ centered at $o$.

For the submanifold $\bar{f}: \bar{S} \rightarrow M$, suppose that $\bar{f}(0)=f(o)$, for some point $\bar{o} \in \bar{S}$. Let $\bar{f}^{i}=y^{i} \circ \bar{f}$, and suppose that $d \bar{f}^{1} \wedge \cdots \wedge d \bar{f}^{p}(\bar{o}) \neq 0$, so that $\bar{x}=\left(\bar{f}^{1}, \cdots, \bar{f}^{p}\right)$ also defines a local coordinate system in $\bar{S}$ centered at $\bar{o}$.

Proposition 1. Let $r$ be a positive integer. The p-dimensional submanifolds $f$ : $S \rightarrow M$ and $\bar{f}: \bar{S} \rightarrow M$ have contact of at least order $r$ at $o \in S$ and $\bar{o} \in \bar{S}$ if and only if $f(o)=\bar{f}(\bar{o})$ and for every integer $k$ for which $1<k \leqslant r$,

$$
\frac{\partial^{k} f^{a}}{\partial f^{\alpha_{1}} \cdots \partial f^{\alpha_{k}}}(o)=\frac{\partial^{k} \bar{f}^{a}}{\partial \bar{f}^{1} \cdots \partial \bar{f}^{\alpha_{k}}}(\bar{o}) .
$$


Remairk. We have here, presumably, what $\mathrm{H}$. Weyl would call the unfixed parameter notion of contact.

Proof. We shall give here the proof for the cases $r=1$ and $r=2$. The pattern for the proof for any $r$ is then quite apparent.

Using the natural isomorphism for any finite dimensional vector spaces $V$ and $W, L(V, W) \cong V^{*} \otimes W$, where $L(V, W)$ is the space of linear transformations from $V$ to $W$, we have $f_{*}=\Sigma_{i} d f^{i} \otimes \partial / \partial y^{i}$. Furthermore,

$$
d f^{a}=\sum_{\alpha} \frac{\partial f^{a}}{\partial f^{\alpha}} d f^{\alpha}
$$

Let $u=\left(\partial / \partial y^{1}, \cdots, \partial / \partial y^{m}\right)$ denote the local coordinate frame field in $M$. Then $T_{f}: S \rightarrow G_{m p}(M)=L(M) x_{G L_{m}} G_{m p}$ is given by $T_{f}=\left[u \circ f,\left[{ }^{I}{ }_{x}\right]\right]$, where $I$ denotes the $p \times p$ identity matrix, and $X_{0}: S \rightarrow R^{(m-p) \times p}$ has for its $a \alpha$ entry the function $\partial f^{a} / \partial f^{\alpha}$.

In the same way, if $\bar{X}_{0}: \bar{S} \rightarrow R^{(m-p) \times p}$ has for its $a \alpha$ entry the function $\partial \bar{f}^{a} / \partial \bar{f}^{\alpha}$, then $T_{\bar{f}}=\left[u \circ \bar{f},\left[{ }_{\bar{x}_{0}}^{I}\right]\right.$. Hence $T_{f}(o)=T_{\bar{f}}(\bar{o})$ if and only if $f(o)=\bar{f}(\bar{o})$ and $X_{0}(o)=\bar{X}_{0}(\bar{o})$; i.e., $f(o)=\bar{f}(\bar{o})$ and $\partial f^{a} / \partial f^{\alpha}(o)=\partial \bar{f}^{a} / \partial \bar{f}^{\alpha}(\bar{o})$ for all $a$ and $\alpha$. This is the desired conclusion for the case $r=1$.

Consider now the case $r=2$. Let $y_{o}=\left(x_{\alpha}^{a}\right): G_{m, p} \supseteq U_{0} \rightarrow \mathbf{R}^{(m-p) \times p}$ denote the standard coordinate system in $G_{m, p}$, and let $\pi: L(M) \rightarrow M$ denote the bundle projection map. Then $\left(y \circ \pi, y_{o}\right)$ defines a local coordinate system in $M^{1}=L(M) x_{G L_{m}} G_{m, p}$ in a neighborhood of points $\left[u,\left[\begin{array}{l}I \\ 0\end{array}\right]\right.$, where $u=$ $\left(\partial / \partial y^{1}, \cdots, \partial / \partial y^{m}\right)$ is the coordinate frame field of the local coordinate system $\dot{y}$ chosen above in $M$.

With respect to the local coordinates $\left(y \circ \pi, y_{o}\right)$, the local representation of $T_{f}: S \rightarrow M^{1}$ is $T_{f}=\left(y \circ f, X_{0}\right)$; (that is, $y \circ \pi \circ T_{f}=y \circ f$ and $\left.y_{o} \circ T_{f}=X_{0}\right)$.

Let $u_{o}=\left(\partial / \partial x_{\alpha}^{a}\right)$ denote the coordinate frame field of $y_{o}$ in $G_{m, p}$. Then

$$
\left(T_{f}\right)_{*}=\sum_{i} d f^{i} \otimes \frac{\partial}{\partial y^{i}}+\sum_{a, \alpha} d\left(\frac{\partial f^{a}}{\partial f^{\alpha}}\right) \otimes \frac{\partial}{\partial x_{\alpha}^{a}} .
$$

Thus $T_{f}^{2}=T_{T_{f}}: S \rightarrow L\left(G_{m, p}(M)\right) x_{G L_{m_{1}}} G_{m_{1} p}$ is given by

$$
T_{f}^{2}=\left[\left(u \circ f, u_{o} \circ X_{0}\right),\left[\begin{array}{l}
I \\
X_{0} \\
X_{1}
\end{array}\right]\right],
$$

where $X_{\mathrm{i}}: S \rightarrow \mathbf{R}^{(m-p) p \times p}$ has for its $(a \alpha, \beta)$ entry the function $\partial^{2} f^{a} / \partial f^{\beta} \partial f^{\alpha}$.

In the same way there is a similar expression for $T_{f}^{2}$, the only difference being that $f, X_{0}$ and $X_{1}$ are replaced by $\bar{f}, \bar{X}_{0}, \bar{X}_{1}$, and $\bar{X}_{1}: \bar{S} \rightarrow \mathbf{R}^{(m-p) p \times p}$ has for its $(a \alpha, \beta)$ entry the function $\partial^{2} \bar{f}^{a} / \partial \bar{f}^{\beta} \partial \bar{f}^{\alpha}$. 
Hence $T_{f}^{2}(o)=T_{\bar{f}}^{2}(o)$ iff $X_{0}(o)=\bar{X}_{0}(\bar{o})$ and $X_{1}(o)=\bar{X}_{1}(\bar{o})$; i.e., iff

$$
\frac{\partial f^{a}}{\partial f^{\alpha}}(o)=\frac{\partial \bar{f}^{a}}{\partial \bar{f}^{\alpha}}(\bar{o}) \text { and } \frac{\partial^{2} f^{\alpha}}{\partial f^{\beta} \partial f^{\alpha}}(o)=\frac{\partial^{2} \bar{f}^{a}}{\partial \bar{f}^{\beta} \partial \bar{f}^{\alpha}}(\bar{o})
$$

for all $a, \alpha, \beta$. This is the desired conclusion for the case $r=2$. q.e.d.

Let $G$ denote any set of diffeomorphisms (local or global) on $M$. In what follows we shall assume that $G$ is a Lie transformation group on $M$, but the concepts defined here make sense when $G$ is, for example, a pseudo-group of local diffeomorphisms of $\boldsymbol{M}$.

Definition. Let $r$ be a nonnegative integer. The $p$-dimensional submanifolds $f: S \rightarrow M$ and $\bar{f}: \bar{S} \rightarrow M$ have $G$-contact of at least order $r$ at $o \in S$ and $\bar{o} \in \bar{S}$ if there exists a transformation $A \in G$ such that $f$ and $A \circ \bar{f}$ have contact of at least order $r$ at $o \in S$ and $\bar{o} \in \bar{S}$.

A diffeomorphism $A$ of $M$ induces a diffeomorphism $A^{(1)}$ of $M^{1}=$ $G_{m, p}(M)$, which sends the tangent $p$-plane $P$ at $x \in M$ to the tangent $p$-plane $A_{*} P$ as $A(x) \in M$. It is easily seen that $A^{(1)}: M^{1} \rightarrow M^{1}$ is smooth, $1: 1$, and onto, and the same is true for its inverse which is $\left(A^{-1}\right)^{(1)}$. With respect to the representation $M^{1}=L(M) x_{G L(m ; R)} G_{m,}$, we have $A^{(1)}[u,[X]]=\left[A_{*} u,[X]\right]$.

Proceeding inductively, $A$ gives rise to a sequence of diffeomorphisms $A^{(R)}$ : $M^{r} \rightarrow M^{r}$ defined by $A^{(R)}=\left(A^{(r-1)}\right)^{(1)}$, for any integer $r \geqslant 1$. (Here $A^{(0)}=$ A.)

Lemma 1. If $f: S \rightarrow M$ is a p-dimensional submanifold of $M$, and $A$ is a diffeomorphism of $M$, then for any positive integer $r, T_{A \circ f}^{r}=A^{(\mathrm{R})} \circ T_{f}^{r}$.

The proof is a straightforward induction.

Suppose again that $f: S \rightarrow M$ and $\bar{f}: \bar{S} \rightarrow M$ are $p$-dimensional submanifolds, and suppose that $G$ is a Lie transformation group on $M$. By Lemma $1, f$ and $\bar{f}$ have $G$-contact of at least order $r$ at $o \in S$ and $\bar{o} \in \bar{S}$ if and only if there exists a transformation $A \in G$ such that $A^{(r)} T_{f}^{r}(\bar{o})=T_{f}^{r}(o)$. But this just means that $T_{\bar{f}}^{r}(\bar{o})$ and $T_{f}^{r}(o)$ both lie in the same orbit of the action of $G$ on $M^{r}$.

The construction of $r$ th-order frames on a submanifold amounts to choosing a cross section for the action of $G$ on $M^{r}$. When $G$ is a Lie transformation group on $M$ the construction is algebraic and depends only on the structure of $G$.

\section{Agreement and deformation}

Definition. Submanifolds $f, \bar{f}: S \rightarrow M$ of dimension $p<m=\operatorname{dim} M$ agree to at least order $r$ at $o \in S$ if they have the same $r$ th order Taylor polynomials at $o$. 
To be more explicit, let $y=\left(y^{1}, \cdots, y^{m}\right)$ be a local coordinate system in $M$ about $f(o)$, and let $x=\left(x^{1}, \cdots, x^{p}\right)$ be a local coordinate system in $S$ about $o$. Let $f^{i}=y^{i} \circ f$ and $\bar{f}^{i}=y^{i} \circ \bar{f}$. Then $f$ and $\bar{f}$ agree to at least order $r$ at $o$ if $f(o)=\bar{f}(o)$ and for any integer $k, 1 \leqslant k \leqslant r,\left(\partial^{k} f^{i} / \partial x^{\alpha_{1}} \cdots \partial x^{\alpha_{k}}\right)(o)$ $=\left(\partial^{k} \bar{f}^{i} / \partial x^{\alpha_{1}} \cdots \partial x^{\alpha_{k}}\right)(o)$, for all $\alpha_{1}, \cdots, \alpha_{k}$ and $i$.

We may assume (reindexing the $y^{i}$ if necessary) that $d f^{1} \wedge \cdots \wedge d f^{p}(o) \neq$ 0 , so that $x=\left(f^{1}, \cdots, f^{p}\right)$ defines a local coordinate system in $S$ centered at $o$. We assume further that $\bar{x}=\left(\bar{f}^{1}, \cdots, \bar{f}^{p}\right)$ also defines a local coordinate system in $S$ about $o$.

Proposition 2. Let $r$ be a positive integer. Submanifolds $f$ and $\bar{f}$ agree at o to at least order $r$ if and only if

(i) they have contact of at least order $r$ at $o$; and

(ii) $\left(\partial \bar{f}^{\alpha} / \partial f^{\beta}\right)(o)=\delta_{\beta}^{\alpha}$ and, for any integer $k$ such that $2<k<r$, $\left(\partial^{k} \bar{f}^{\alpha} / \partial f^{\beta_{1}} \ldots \partial f^{\beta_{k}}\right)(o)=0$.

Remark. Conditions (ii) are equivalent to the condition that the local diffeomorphism $\bar{x} \circ x^{-1}$ of $R^{p}$ agree with the identity transformation to at least order $r$ at the origin.

Proof. An elementary application of Proposition 1 and the chain rule. q.e.d.

Let $f: S \rightarrow M$ and $\bar{f}: \bar{S} \rightarrow M$ be $p$-dimensional submanifolds of $M$. Let $G$ be a Lie transportation group on $M$, and $r$ a positive integer.

Definition. $f$ and $\bar{f}$ are $r$ th order $G$-deformations of each other if there exist a smooth map $v: S \rightarrow G$ and a diffeomorphism $F: S \rightarrow \bar{S}$ such that, for each point $s \in S, f$ agrees to at least order $r$ with $v(s) \circ \bar{f} \circ F$ at $s$.

The deformation is trivial if $f$ and $\bar{f}$ are actually $G$-congruent; that is, if $v(s)$ can be chosen so that it does not depend on $s \in S$. We shall say that $f$ is rigid with respect to $r$ th-order $G$-deformations if there are no nontrivial $r$ th-order $G$-deformations of it.

Remark. Deformation implies contact. To be precise, if $f$ and $\bar{f}$ are $r$ th-order $G$-deformations of each other, then there exists a diffeomorphism $F: S \rightarrow \bar{S}$ such that $f$ and $\bar{f} \circ F$ have $G$-contact of at least order $r$ at every point of $S$.

Example. The notion of deformation lies behind many of the concepts and problems of classical differential geometry. Consider, for example, the case of surfaces in Euclidean space. Then $M=\mathbf{R}^{3}$ on which acts the group $G=E(3)$ of proper rigid motions. Let $d s^{2}$ denote the Euclidean metric on $\mathbf{R}^{3}$.

Two surfaces $f: S \rightarrow \mathbf{R}^{3}$ and $\bar{f}: \bar{S} \rightarrow \mathbf{R}^{3}$ are first order $E(3)$-deformations of each other if and only if they are isometric with respect to their induced metrics. In other words, there are a smooth map $v: S \rightarrow E(3)$ and a diffeomorphism $F: S \rightarrow \bar{S}$ such that $f$ and $v(s) \circ \bar{f} \circ F$ agree to first order at $s$, for 
every $s \in S$, if and only if there exists a diffeomorphism $F: S \rightarrow \bar{S}$ such that $F^{*} \bar{f}^{*} d s^{2}=f^{*} d s^{2}$. (Of course this is local; $S$ and $\bar{S}$ may have to be shrunk.)

It is well-known that locally any surface in $\mathbf{R}^{3}$ has nontrivial first order $E(3)$-deformations, and that the deformations of a given surface are given by the arbitrary choice of two functions of one variable (cf. [4, pp. 141-144]). Using the Riemannian connection and Gaussian curvature of the induced metric, one can give simple necessary and sufficient conditions for when two surfaces are first order $E$ (3)-deformations of each other. (Cf. [3, pp. 227-230]. In this account of Cartan's there are certain regularity assumptions tacitly made. For example, he did not characterize the deformations of a neighborhood of an isolated critical point of the Gaussian curvature. To my knowledge that remains an open problem.)

Finally, it is a celebrated theorem of Gauss that two surfaces are second order $E(3)$-deformations of each other if and only if they are $E(3)$-congruent. That is, all surfaces have second order rigidity.

The problem of finding all surfaces in $\mathbf{R}^{3}$ isometric to a given surface in $\mathbf{R}^{3}$ was considered by Gauss in [5, p. 45]. It is interesting to notice that Gauss formulated this problem in terms of deformation. It seems quite possible that Gauss's study [5] was guided by the general philosophy of determining quantities, for example distance and curvature, on a surface which are invariant under first order $E(3)$-deformations.

\section{The differential system of a deformation}

We consider $k$ th order deformations of $p$-dimensional submanifolds of a homogeneous space $M=G / G_{0}$, where $k$ and $p$ are integers, $k \geqslant 1$, and $1 \leqslant p \leqslant n=\operatorname{dim} M$.

To summarize the method, the differential equations of a deformation are obtained by expanding in Taylor polynomials the two submanifolds with respect to any adapted frame of $M$; i.e., elements of $G$. Keeping a point of the submanifold fixed, but varying the point of $G$, we differentiate these Taylor polynomials and equate coefficients of like monomials up to degree $k$.

The method begins with a once and for all choice of real analytic local coordinate system in $M$ centered at $o \in M$. It is convenient for us to write down its inverse, a local parametrization $(\mathcal{Q}, \varphi)$, where $\mathscr{U}$ is a neighborhood of $0 \in \mathbf{R}^{n}$, and $\varphi: \mathcal{Q} \rightarrow M$ maps $\mathcal{Q}$ diffeomorphically onto a neighborhood of $o$ in $M$ such that $\varphi(0)=o$. For any $u \in G,(\mathcal{Q}, u \circ \varphi)$ is a local parametrization of $M$ centered at $u(o)$.

Let $P: G \times \mathscr{Q} \rightarrow M$ be defined by $P(u, x)=u(\varphi(x))$. Notice that for each fixed $u \in G$, the map $P_{u}(x)=P(u, x)=u \circ \varphi(x)$ maps $Q$ diffeomorphically 
onto $u(\varphi(M)) \subseteq M$. For each $u \in G$ and $p \in u(\varphi(थ))$, we shall call

$$
x=\left[\begin{array}{c}
x^{1} \\
\vdots \\
x^{n}
\end{array}\right)=(u \circ \varphi)^{-1}(p)
$$

the coordinates of $p$ with respect to the frame $u$.

Proposition 2. For any point $p \in M, P^{-1}\{p\}$ is a codimension-n real analytic submanifold of $G \times$ थ whose tangent space at any point is defined by the $n$ linear equations

$$
d x^{i}=\sum_{\sigma} A_{\sigma}^{i} \Omega^{\sigma}, \quad 1 \leqslant i \leqslant n, 1 \leqslant \sigma \leqslant \operatorname{dim} G .
$$

Here $\left\{\Omega^{\sigma}\right\}$ is a basis of left-invariant 1-forms on $G$, the $A_{\sigma}^{i}$ are real analytic functions on $\mathcal{U}$, restricted to $P^{-1}\{p\}$, and the $x^{i}$ are the standard coordinate functions on $R^{n}$.

Proof. That $P^{-1}\{p\}$ is a codimension- $n$ submanifold whose tangent space is defined by $n$ linear equations involving no relations among $d x^{1}, \cdots, d x^{n}$ follows from the fact that at any $(u, x) \in G \times \mathcal{U}, P_{*}(G \times \mathcal{U})_{(u, x)} \supseteq P_{u_{*}} \mathcal{U}_{x}$ $=M_{P(u, x)}$. Hence if $(0, \xi) \in\left(P^{-1}\{p\}\right)_{(u, x)}$, then $0=P_{*}(0, \xi)=P_{u_{*}} \xi$, so $\xi=0$. It remains to show that the $A_{o}^{i}$ are real analytic functions on $\mathcal{Q}$ (restricted to $\left.P^{-1}\{p\}\right)$.

Varying $p$ over all of $M$ shows that the $A_{\sigma}^{i}$ in (4.1) are the restriction to $P^{-1}\{p\}$ of functions $A_{\sigma}^{i}(u, x)$ on $G \times \mathcal{U}$. For any $a \in G$, define $L_{a}: G \times \mathcal{U}$ $\rightarrow G \times \mathscr{U}$ by $L_{a}(u, x)=(a u, x)$. The tangent space to $P^{-1}\{P(u, x)\}$ at $(u, x)$ is given by

$$
d x^{i}(x)=\sum_{\sigma} A_{\sigma}^{i}(u, x) \Omega^{\sigma}(u)
$$

while the tangent space to $P^{-1}\{P(a u, x)\}$ at $(a u, x)$ is given by

$$
d x^{i}(x)=\sum_{\sigma} A_{\sigma}^{i}(a u, x) \Omega_{(a u)}^{\sigma}
$$

But $P\left(L_{a}(u, x)\right)=P(a u, x)=(a u)(\varphi(x))=a(u(\varphi(x)))=a P(u, x)$ implies that $L_{a}\left(P^{-1}\{P(u, x)\}\right)=P^{-1}\{a P(u, x)\}$. Thus the tangent space to $P^{-1}\{P(u, x)\}$ at $(u, x)$ is given by

$$
L_{a}^{*}\left(d x^{i}(x)=\sum_{\sigma} A_{\sigma}^{i}(a u, x) \Omega_{(a u)}^{\sigma}\right)
$$

But $L_{a}^{*} d x_{(x)}^{i}=d x_{(x)}^{i}$, and $L_{a}^{*} \Omega_{(a u)}^{\sigma}=\Omega_{(u)}^{\sigma}$. Comparing (4.2) and (4.3) it follows that $A_{\sigma}^{i}(a u, x)=A_{\sigma}^{i}(u, x)$, for every $a \in G$. Hence $A_{\sigma}^{i}(u, x)$ does not depend on $u$; it is a function of $x$ alone. q.e.d.

We shall derive (4.1) for two classes of examples. 
Example 1. $M=\mathbf{R}^{n}=G / G_{0}$, where $G$ is any subgroup of the affine group which acts transitively on $\mathbf{R}^{n}$. (For example, $G$ is the whole affine group, or the equiaffine group, or the conformal affine group, or the Euclidean proper motion group.)

The affine group is a semi-direct product $G L(n ; \mathbf{R}) \cdot \mathbf{R}^{n}$, and its Lie algebra is a semi-direct sum $\operatorname{SII}(n ; \mathbf{R})+\mathbf{R}^{n}$. Its Maurer-Cartan form $\Omega$ is the $\operatorname{SI}(n ; \mathbf{R})$ $+\mathbf{R}^{n}$ valued left-invariant 1-form:

$$
\Omega_{(A, q)}=\left(A^{-1} d A, A^{-1} d q\right), \quad A \in G L(n ; \mathbf{R}), x \in \mathbf{R}^{n} .
$$

Put $\left(\Omega_{j}^{i}\right)=A^{-1} d A$ and $\left(\Omega^{i}\right)=A^{-1} d x, 1 \leqslant i, j \leqslant n$. Then $\left\{\Omega_{j}^{i}\right.$, $\left.\Omega^{i}\right\}$ is a basis of left-invariant 1 -forms on the affine group. Some subset forms a basis of left-invariant 1-forms on $G$.

Our choice of parametrization $\varphi$ will be the identity map $U=\mathbf{R}^{n} \rightarrow$ $\mathbf{R}^{n}$. Then $P: G \times \mathbf{R}^{n} \rightarrow \mathbf{R}^{n}$ is given by $P(u, x)=u(x)$. Any $u \in G \subset$ $G L(n ; \mathbf{R}) \cdot \mathbf{R}^{n}$ can be written uniquely as $u=(A, q)$, for some $A \in$ $G L(n ; R), q \in R^{n}$. Put $A=\left(e_{1}, \cdots, e_{n}\right)$, where $e_{i}=i$ th column of $A$. Then $P(u, x)=u(x)=q+A x=q+\sum_{i} x^{i} e_{i}$, where $x=\left(x^{i}\right)$.

If $p=P(u, x)=q+\sum_{i} x^{i} e_{i}$ is fixed, then

$$
0=d p=d q+\sum_{i} d x^{i} e_{i}+\sum_{i} x^{i} d e_{i} \text {. }
$$

Since $d q=\Sigma_{j} \Omega^{j} e_{j}$ and $d e_{i}=\sum_{j} \Omega_{i}^{j} e_{j}$ by (4.4), we can substitute this into (4.5) and get

$$
0=\sum_{i}\left(\Omega^{i}+d x^{i}+\sum_{j} x^{j} \Omega_{j}^{i}\right) e_{i}
$$

Hence

$$
d x^{i}=-\Omega^{i}-\sum_{j} x^{j} \Omega_{j}^{i}
$$

These are the equations (4.1) for this space.

Example 2. Grassmannians. $M=G_{n, p}$, the space of all oriented $p$-dimensional linear subspaces of $R^{n} 1 \leqslant p \leqslant n-1$. For $G$ we take any subgroup of $G L(n ; \mathbf{R})$ which acts transitively on $G_{n, p}$. The most important cases would be $G=S L(n ; \mathbf{R})$, the special linear group; or $G=O(n)$, the orthogonal group.

The Maurer-Cartan form of $G L(n ; \mathbf{R})$ is $\Omega=A^{-1} d A=\left(\omega_{j}^{i}\right), 1<i, j<n$. Thus $\left\{\Omega_{j}^{i}: 1 \leqslant i, j \leqslant n\right\}$ is a basis of left-invariant 1 -forms on $G L(n ; \mathbf{R})$. Some subset is a basis of such forms on $G$. We shall use the following index convention:

$$
1 \leqslant i, j \leqslant n ; \quad 1 \leqslant \alpha, \beta \leqslant p ; \quad p+1 \leqslant a, b \leqslant n .
$$


Our choice of local parametrization of $G_{n, p}$ is $\varphi: \mathbf{R}^{(n-p) \times p} \rightarrow G_{n, p}$ given by $\varphi(X)=\left[{ }_{X}^{I}\right]$, (we are following the notation of $\S 1$ ). Then $P: G \times \mathbf{R}^{(n-p) \times p} \rightarrow$ $G_{n, p}$ is $P(u, X)=u \cdot\left[\begin{array}{l}I \\ X\end{array}\right]=\left[u \cdot\left(\begin{array}{l}I \\ X\end{array}\right)\right]$.

If $\pi: \tilde{\mathbf{R}}^{n \times p} \rightarrow G_{n, p}$ denotes the canonical projection of an $n \times p$ matrix $X$ of rank $p$ to its equivalence class $[X] \in G_{n, p}$, then $P$ factors into $P=\pi \circ \tilde{P}$, where $\tilde{P}: G \times \mathbf{R}^{(n-p) \times p} \rightarrow \tilde{\mathbf{R}}^{n \times p}$ is given by $\tilde{P}(u, X)=u \cdot\left(\begin{array}{l}I \\ X\end{array}\right)$.

Now $P$ is constant if and only if $\tilde{P}$ lies in $\{\tilde{P} \cdot A: A \in G L(p ; \mathbf{R})\}$. Thus on a submanifold $P=$ constant,

$$
d \tilde{P}=\tilde{P} \Lambda,
$$

where $\Lambda$ is a $p \times p$ matrix of 1 -forms on $G \times \mathbf{R}^{(n-p) \times p}$, which we denote $\Lambda=\left(\Lambda_{\beta}^{\alpha}\right)$.

On the other hand, if we write, for $u \in G, u=\left(e_{1}, \cdots, e_{n}\right)$, where $e_{i}$ is the $i$ th column of $u$; and if we write $X=\left(x_{\alpha}^{a}\right) \in \mathbf{R}^{(n-p) \times p}$, then

$$
\tilde{P}=u \cdot\left(\begin{array}{l}
I \\
X
\end{array}\right)=u \cdot\left(\left(\begin{array}{l}
I \\
0
\end{array}\right)+\left(\begin{array}{l}
0 \\
X
\end{array}\right)\right)=\left(e_{1}, \cdots, e_{p}\right)+\left(e_{a} x_{1}^{a}, \cdots, e_{a} x_{p}^{a}\right),
$$

(sum on repeated indices). Thus $d \tilde{P}=\left(d e_{1}, \cdots, d e_{p}\right)+\left(\cdots, d e_{a} x_{\alpha}^{a}+\right.$ $\left.e_{a} d x_{\alpha}^{a}, \cdots\right)$, so

$$
d \tilde{P}=\left(\cdots, \Omega_{\alpha}^{i} e_{i}, \cdots\right)+\left(\cdots, \Omega_{a}^{i} x_{\alpha}^{a} e_{i}+e_{a} d x_{\alpha}^{a}, \cdots\right),
$$

since $d e_{i}=\Omega_{i}^{j} e_{j}$.

Comparing the coefficients of $e_{\alpha}$ in (4.7) and (4.8),

$$
\Lambda_{\beta}^{\alpha}=\Omega_{\beta}^{\alpha}+\Omega_{b}^{\alpha} x_{\beta}^{b} .
$$

Comparing the coefficients of $e_{a}$ in (4.7) and (4.8),

$$
x_{\alpha}^{a} \Lambda_{\beta}^{\alpha}=\Omega_{\beta}^{a}+\Omega_{b}^{a} x_{\beta}^{b}+d x_{\beta}^{a} .
$$

Eliminating the $\Lambda_{\beta}^{\alpha}$ from (4.9) and (4.10), we get (4.1) for this space:

$$
d x_{\beta}^{a}=-\Omega_{\beta}^{a}+x_{\alpha}^{a} \Omega_{\beta}^{\alpha}-\Omega_{b}^{a} x_{\beta}^{b}+x_{\alpha}^{a} \Omega_{b}^{\alpha} x_{\beta}^{b} .
$$

In the case $G=G L(n ; \mathbf{R})$, the set of forms $\left\{\Omega_{j}^{i}\right\}$ is a basis of left-invariant 1 -forms on $G$. If $G$ is a subgroup of $G L(n ; \mathbf{R})$, then some subset of $\left\{\Omega_{j}^{i}\right\}$ forms a basis of $G$, the remaining forms being linear combinations of these.

We continue now with the derivation of the differential equations of a $k$ th order deformation. Suppose that the $p$-dimensional submanifolds $f: S \rightarrow M$ and $\bar{f}: \bar{S} \rightarrow M$ are $k$ th order deformations of each other. Then there are a smooth map $v: S \rightarrow G$ and a diffeomorphism $F: S \rightarrow \bar{S}$ such that at each $s \in S, v(s) \circ \bar{f} \circ F$ agrees through order $k$ with $f$ at $s$. Replacing $\bar{f}$ by $\bar{f} \circ F$, we may as well assume that $\bar{S}=S$ and that $F=$ identity transformation. 
For each $(s, t) \in S \times S$, the coordinates of $f(t)$ with respect to $u(s)$ are the components $x^{1}, \cdots, x^{n}$ of $x(s, t) \in \mathbf{R}^{n}$ given by $x: S \times S \rightarrow \mathbf{R}^{n}$,

$$
x(\alpha, t)=(u(s) \circ \varphi)^{-1}(f(t))
$$

Without loss of generality we may assume that for each $s \in S$, the functions $x^{1}, \cdots, x^{p}$ define local coordinates in $S$ centered at $s$.

The coordinates of $\bar{f}(t)$ with respect to $\bar{u}(s)$ are given by the components $\bar{x}^{i}$ of

$$
\bar{x}(s, t)=(\bar{u}(s) \circ \varphi)^{-1}(\bar{f}(t)) .
$$

However, since $\bar{u}(s)=v(s)^{-1} u(s)$,

$$
\bar{x}(s, t)=\left(v(s)^{-1} \cdot u(s) \circ \varphi\right)^{-1}(\bar{f}(t))=(u(s) \circ \varphi)^{-1}(v(s) \circ \bar{f}(t)) .
$$

Thus $\bar{x}(s, t)$ is also the coordinate of $v(s) \circ \bar{f}(t)$ with respect to $u(s)$.

The condition that $v(s) \circ \bar{f}$ agrees through order $k$ with $f$ at $s$ can be expressed as, for each $s$,

$$
\begin{aligned}
\bar{x}^{i}\left(s ; x^{1}, \cdots,\right. & \left.x^{p}\right)=x^{i}\left(s ; x^{1}, \cdots, x^{p}\right), \\
& \text { (modulo terms of degree } \left.>k \text { in } x^{1}, \cdots, x^{p}\right) .
\end{aligned}
$$

Differentiating (4.12) with respect to $s$, keeping $t$ fixed:

$$
\left.d \bar{x}^{i}=d x^{i} \quad \text { (modulo terms of degree }>k \text { in } x^{1}, \cdots, x^{p}\right) .
$$

Put $\omega^{\sigma}=u^{*} \Omega^{\sigma}$ and $\bar{\omega}^{\sigma}=\bar{u} \Omega^{\sigma}, 1$-forms on $S$, and let id: $\mathbf{R}^{n} \rightarrow \mathbf{R}^{n}$ denote the identity map, then applying $(u \times \mathrm{id})^{*}$ and $(\bar{u} \times \mathrm{id})^{*}$ to $(4.1)$ gives

$$
d x^{i}=\sum_{\sigma} A_{\sigma}^{i}(x) \omega^{\sigma}, \quad d \bar{x}^{i}=\sum_{\sigma} A_{\sigma}^{i}(\bar{x}) \bar{\omega}^{\sigma} .
$$

Putting these relations into (4.13) gives

$$
\sum_{\sigma} A_{\sigma}^{i}(\bar{x}) \bar{\omega}^{\sigma}=\sum_{\sigma} A_{\sigma}^{i}(x) \omega^{\sigma} \quad \text { (modulo terms of degree }>k \text { ). }
$$

In (4.14) replace $A_{\sigma}^{i}$ by its Taylor polynomial of order $k-1$ in $x^{i}, \cdots, x^{n}$; then replace $x^{p+1}, \cdots, x^{n}$ by their Taylor polynomials in $x^{1}, \cdots, x^{p}$ of order $k-1$; and finally replace each $\bar{x}^{i}$ by $x^{i}$ because of (4.12). Thus, modulo terms in $x^{1}, \cdots, x^{p}$ of order $>k$, we can replace $A_{\sigma}^{i}(\bar{x})$ and $A_{\sigma}^{i}(x)$ by their $(k-1)$ th order Taylor polynomial in $x^{1}, \cdots, x^{p}$ :

$$
A_{\sigma}^{i}=\sum_{0<|I|<k} C_{\sigma I}^{i} x^{I}
$$

where $x=\left(x^{i}, \cdots, x^{p}\right), I=\left(i_{1}, \cdots, i_{p}\right)$, and the $C_{\sigma I}^{i}$ are functions of $s$. Equating coefficients of each $x^{I}$ gives the exterior differential equations of a $k$ th order $G$-deformation:

$$
C_{\sigma I}^{i}\left(\bar{\omega}^{\sigma}-\omega^{\sigma}\right)=0, \quad i=1, \cdots, n, \sigma=1, \cdots, \operatorname{dim} g, 0 \leqslant|I|<k .
$$

They are a necessary condition for local $k$ th order $G$-deformation. 
These differential equations (4.16) give a sufficient condition as well for local $k$ th order deformation. To be precise, suppose $f, \bar{f}: S \rightarrow M$ are $p$-dimensional submanifolds with frame fields $u, \bar{u}: S \rightarrow G$, respectively. If $x(s, t)=$ $(u(s) \circ \varphi)^{-1} f(t)$ and $\bar{x}(s, t)=(\bar{u}(s) \circ \varphi)^{-1} \bar{f}(t)$, satisfy (4.16), with $\omega^{\sigma}=u^{*} \Omega^{\sigma}$ and $\bar{\omega}^{\sigma}=\bar{u}^{*} \Omega^{\sigma}$ as above, then $\bar{f}$ is a $k$ th order deformation of $f$. Furthermore the deformation is achieved by $v: S \rightarrow G$ given by $v(s)=u(s) \bar{u}(s)^{-1}$.

\section{Solving the deformation problems}

The exterior differential system (4.16) derived in $\$ 4$ gives necessary and sufficient conditions for two $p$-dimensional submanifolds to be $k$ th order $G$-deformations of each other. Consider now the following three questions:

(1) Given $k$ and $p$, are there any nontrivial $k$ th order $G$-deformations of $p$-dimensional submanifolds of $M$ ?

(2) How does one find all $k$ th order $G$-deformations of a given submanifold?

(3) How does one recognize whether or not two given $p$-dimensional submanifolds are $k$ th order $G$-deformations of each other?

General methods for answering these questions are outlined in this section. These methods will be described in detail below for equiaffine deformations of surfaces in 3-space. This section concludes with the result that under quite general conditions all submanifolds of $M$ are rigid to sufficiently high order $G$-deformations.

The methods used here make use of the higher order contact frames constructed in [7]. For a $p$-dimensional submanifold $f: S \rightarrow M$, the $k$ th order frames on $f$ are denoted by $L_{k}$, which is a submanifold of $G$, and $L_{k} \rightarrow S$ is a fibration whose fiber at each point is a coset of a subgroup of the isotropy subgroup $G_{0}$. For example, $L_{0}=\{u \in G: u(o) \in f(S)\}$, whose fiber $u$ is $u \cdot G_{0}$. Then $L_{0} \supseteq L_{1} \supseteq L_{2} \supseteq \cdots \cdot$

Continue to let $\left\{\Omega^{\sigma}\right\}$ denote a basis of left-invariant 1-forms on $G$. In the construction of $L_{1}$, there are $p$ of these forms - which we may as well assume to be $\Omega^{1}, \cdots, \Omega^{p}$ - which pull back by any cross section $u: S \rightarrow L_{1}$ to a coframe field on $S$. Furthermore this is true for any cross section $u: S \rightarrow L_{k}$, $k \geqslant 1$. Finally, each $L_{k}$ is an integral submanifold of $G$ of an exterior differential system on $G$.

The method for answering question (1) is to consider the exterior differential system on $G \times G$ consisting of (4.16) (with $\omega^{\sigma}, \bar{\omega}^{\sigma}$ replaced by $\Omega^{\sigma}$ and $\bar{\Omega}^{\circ}$, respectively) together with the equations of the systems defining $L_{k} \subseteq G$ and $\bar{L}_{k} \subseteq G$. (Here we let $\left\{\bar{\Omega}^{\sigma}\right\}$ denote the same basis of left-invariant forms, but 
on the second copy of $G$.) This system is to have designated independent variables $\Omega^{1}, \cdots, \Omega^{p}$. Then any $p$-dimensional integral submanifold of it will be given by $(u, \bar{u}): S \rightarrow G \times G$, and it will follow that $f=\pi \circ u$ and $\bar{f}=\pi \circ \bar{u}$ are $k$ th order $G$-deformations of each other. (Here $\pi: G \rightarrow M$ is the projection $\pi(u)=u(o)$.)

For question (2), let $f: S \rightarrow M$ be a given submanifold of constant $k$ th order type, and let $L_{k}$ be its $k$ th order frames. Let $i: L_{k} \rightarrow G$ be the inclusion mapping, and let $\omega^{\sigma}=i^{*} \Omega^{\sigma}$. Then only a subset of $\left\{\omega^{\sigma}\right\}$ are linearly independent (but a subset containing $\omega^{1}, \cdots, \omega^{p}$ in every case), the remainder will be linear combinations of forms in the basis subset, with coefficients which are functions on $L_{k}$.

To find all $k$ th order $G$-deformations of $f$, find all $p$-dimensional integral submanifolds of the exterior differential system on $L_{k} \times G$ defined by (4.16), (where $\bar{\omega}^{\sigma}$ is replaced by $\Omega^{\sigma}$ ), together with the equations on the $\Omega^{\sigma}$ which define the $k$ th order frames $L_{k}$. Again $\omega^{1}, \cdots, \omega^{p}$ are the designated independent variables. A p-dimensional integral submanifold $(u, u): S \rightarrow L_{k}$ $\times G$ will give a pair of submanifolds which are $k$ th order $G$-deformations of each other, and one of them is $\pi \circ u=f$, the given submanifold.

If this system on $L_{k} \times G$ is in involution, then a general $f$ possesses many nontrivial deformations. If the system is completely integrable, then any $f$ is rigid. If the system is not in involution, then generic $f$ are rigid, but by the method of prolongation, necessary conditions, on $f$ can be obtained for the existence of nontrivial deformations.

Finally, to answer question (3), one looks for functions on the submanifold which are invariant under $k$ th order $G$-deformations. Always the contact invariants of orders up through (and sometimes even higher ordeR), will be invariant under $k$ th order $G$-deformations, but sometimes there are none, or not enough. Even so, functions can be constructed sometimes from higher order contact invariants which can be used. For example, for surfaces in Euclidean space, every surface possesses nontrivial 1st order rigid motion deformations. However, there are no first order contact invariants. Nevertheless, from the two second order contact invariants, the principal curvatures, the Gaussian curvature can be constructed, and it is invariant under 1st order deformation even though the principal curvatures are not.

The following result resolves $[6,15.1$, p. 799]

Theorem. Let $M=G / G_{0}$ be a homogeneous space for which the frame construction of [7] can be carried out. Then there is an order $k \geqslant 1$ such that any submanifold of constant type is rigid to kth order G-deformations.

Proof. If $f, \bar{f}: S \rightarrow M$ are $k$ th order $G$-deformations of each other, then for each $s \in S, f$ and $\bar{f}$ have $k$ th order $G$-contact at $s$. By [7, Theorem 3, p. 32] if $f$ 
is of constant type for $k$ sufficiently large, then any submanifold $\bar{f}$ which has $k$ th order $G$-contact with $f$ at $s$ for every $s \in S$ is $G$-congruent to $f$.

\section{EQUIAFFINE DEFORMATIONS OF SURFACES}

\section{The equiaffine group}

The equiaffine group $S A(m)$ is the Lie group of all unimodular affine transformations of $\mathbf{R}^{m}$. This group can be represented as the subgroup of $G L(m+1 ; \mathbf{R})$ given by

$$
S A(m)=\left\{(A, x)=\left(\begin{array}{cc}
A & x \\
0 & 1
\end{array}\right): A \in S L(m ; \mathbf{R}), x \in \mathbf{R}^{m}\right\},
$$

where $S L(m ; \mathbf{R})$ is the special linear group, (all $m \times m$ real matrices whose determinant is equal to 1$)$.

The action of $S A(m)$ on $\mathbf{R}^{m}$ is given by

$$
(A, x) y=A y+x,
$$

for any $y \in \mathbf{R}^{m}$. This action is transitive, and the isotropy subgroup at the origin 0 of $\mathbf{R}^{m}$ is $G_{0}=\{(A, 0): A \in S L(m ; \mathbf{R})\} \simeq S L(m ; \mathbf{R})$. For convenience we shall usually denote the element $(A, 0)$ in $G_{0}$ simply by $A$. The projection map $\pi: S A(m) \rightarrow \mathbf{R}^{m}$ is given by $\pi(A, x)=(A, x) 0=x$.

The Lie algebra of $S A(m)$ is represented in $\operatorname{gl}(m+1 ; \mathbf{R})$ by

$$
\mathfrak{g}=\left\{(X, x)=\left(\begin{array}{ll}
X & x \\
0 & 0
\end{array}\right): X \in \operatorname{si}(m ; \mathbf{R}), x \in \mathbf{R}^{m}\right\},
$$

where $\mathfrak{s I}(m ; \mathbf{R})$ is the Lie algebra of all $m \times m$ real matrices whose trace is equal to 0 .

The Lie algebra of $G_{0}$ is the subalgebra of $g$ :

$$
\mathrm{g}_{0}=\left\{\left(\begin{array}{cc}
X & 0 \\
0 & 0
\end{array}\right): X \in \operatorname{sl}(m ; \mathbf{R})\right\} \cong \operatorname{sl}(m ; \mathbf{R}) .
$$

We shall usually let $X$ denote the element $(X, o)$. If we let $\mathfrak{m}_{0}=\{(0, y) \in \mathfrak{g}$ : $\left.y \in \mathbf{R}^{m}\right\}$, then $\mathfrak{m}_{0}$ is a vector space complement to $g_{0}$ in $g$, and the adjoint action of $G_{0}$ on $\mathrm{m}_{0}$ is simply

$$
\operatorname{ad}(A)(0, y)=(0, A y), \quad \text { for } A \in S L(m ; R), x \in R^{m} .
$$

For future reference we point out that the adjoint action of $G_{0}$ on $g$ is given by

$$
\operatorname{ad}(A)(X, y)=\left(A X A^{-1}, A y\right) .
$$

The Maurer-Cartan form of $S A(m)$ is the $g$-valued left-invariant 1-form on $S A(m)$

$$
\Omega_{(A, x)}=\left(A^{-1} d A, A^{-1} d x\right)
$$


We shall write $\Omega=\left(\left(\Omega_{j}^{i}\right),\left(\Omega^{i}\right)\right)$, where $\Omega_{j}^{i}$, $\Omega^{i}$ are ordinary left-invariant 1forms on $S A(m)$ and $\Sigma_{1}^{m} \Omega_{i}^{i}=0$.

The structure equations of $S A(m)$ are contained in the formula $d \Omega=-\Omega \wedge$ $\Omega$, which in detail is the set of equations

$$
\begin{aligned}
& d \Omega_{j}^{i}=-\sum_{k} \Omega_{k}^{i} \wedge \Omega_{j}^{k}, \\
& d \Omega^{i}=-\sum_{k} \Omega_{k}^{i} \wedge \Omega^{k} .
\end{aligned}
$$

We specialize now to the case $m=3$. For the rest of the paper we let $G=S A(3)$. Our index convention becomes:

$$
1 \leqslant i, j, k \leqslant 3 ; \quad 1 \leqslant \alpha, \beta \leqslant 2 .
$$

A linear frame $u$ at a point $x$ of $\mathbf{R}^{3}$ can be denoted $u=\left(e_{1}, e_{2}, e_{3} ; x\right)$, where $e_{1}, e_{2}, e_{3}$ are three linearly independent vectors in $\mathbf{R}^{3}$. As a reference frame $u_{0}$ we choose the standard basis $\varepsilon_{1}, \varepsilon_{2}, \varepsilon_{3}$ at the origin 0 of $\mathbf{R}^{3}$. Then we get a bundle monomorphism $h_{o}: G \rightarrow L\left(\mathbf{R}^{3}\right)$ given by $h_{o}(A, x)=(A, x)_{*} u_{0}=$ $\left(A \varepsilon_{1}, A \varepsilon_{2}, A \varepsilon_{3} ; x\right)$. Thus $h_{0}$ identifies $(A, x) \in G$ with the frame at $x$ given by the three columns of $A$. The associated representation $\rho_{o}: G_{o} \rightarrow G L(3 ; \mathbf{R})$ is just the standard representation of $S L(3 ; \mathbf{R})$. Then $h_{o}((A, x) K)=$ $h_{o}(A, x) \rho_{o}(K)$ for any $(A, x) \in G, K \in G_{o}$.

The frames $h_{o}(G)$ can be uséd to simplify the representation of the Grassmann bundle $G_{3,2}\left(\mathbf{R}^{3}\right)$. In fact, $G_{3,2}\left(\mathbf{R}^{3}\right)=G x_{G_{o}} G_{3,2}$, where we have written $G$ instead of $h_{o}(G)$.

\section{Frames}

Suppose that $f: S \rightarrow \mathbf{R}^{3}$ is a regularly imbedded surface in $\mathbf{R}^{3}$. A zeroth order frame at $s \in S$ is any element $u \in G$ such that $\pi(u)=f(s)$. Then $h_{o}(u)=\left(e_{1}, e_{2}, e_{3} ; f(s)\right)$. We shall usually abuse notation and write $u=$ $\left(e_{1}, e_{2}, e_{3} ; f(s)\right)$.

Definition. A zeroth order frame field along $f$ is a smooth map $u: S \rightarrow G$ such that $\pi \circ u=f$; i.e., such that $u(s)$ is a zeroth order frame of $f$ at $s$ for every $s \in S$.

In general, suppose that $x: S \rightarrow \mathbf{R}^{3}$ is a smooth map, let $s \in S$ and suppose that $v=\left(v_{1}, v_{2}, v_{3}\right)$ is a frame for $\mathbf{R}^{3}$. Then

$$
d x_{(s)}=x_{*(s)}=\sum_{i} \varphi^{i} \otimes v_{i}
$$

where the $\varphi^{i}$ are linear functionals on the tangent space $S_{s}$, called by E. Cartan the relative components of infinitesimal displacement of $x$ at $s$ with 
respect to the frame $v$. Now suppose that $u=\left(e_{1}, e_{2}, e_{3} ; f\right): S \rightarrow G$ is a zeroth order frame field along $f$. Then $e_{1}, e_{2}, e_{3}$ and $f$ are smooth maps $S \rightarrow \mathbf{R}^{3}$, and so their relative components of infinitesimal displacement at any point with respect to the frame $u$ are given by

$$
d e_{i}=\sum_{j} \omega_{i}^{j} \otimes e_{j}, \quad d f=\sum_{j} \theta^{j} \otimes e_{j} .
$$

Lemma 2. If $u=\left(e_{1}, e_{2}, e_{3} ; f\right)$ is a smooth zeroth order frame field along $f$, then the relative components $\theta^{i}$ and $\omega_{j}^{i}$ of infinitesimal displacement of $f$ and $e_{i}$, respectively, defined in (1) are given by

$$
\theta^{i}=u^{*} \Omega^{i}, \quad \omega_{j}^{i}=u^{*} \Omega_{j}^{i} .
$$

Proof. If $(A, a) \in G$ and $(X, x) \in \mathfrak{g}$, and if $L_{(A, a)}$ denotes left multiplication on $G$ by $(A, a)$, then $L_{(A, a)^{*}}(X, x)=(A X, A x)$. Let $A: S \rightarrow S L(3 ; \mathbf{R})$ be the matrix valued function whose $i$ th column is $e_{i}$. Then

$$
\begin{aligned}
u^{*} \Omega_{(s)} & =L_{u(s)^{-1} * u_{*(s)}}=L_{\left(A(s)^{-1},-A(s)^{-1} f(s)\right) *}\left(d e_{1}, d e_{2}, d e_{3} ; d f\right)(s) \\
& =\left(A(s)^{-1} \cdot d e_{1}, A(s)^{-1} \cdot d e_{2}, A(s)^{-1} \cdot d e_{3} ; A(s)^{-1} \cdot d f\right)(s) .
\end{aligned}
$$

Applying $A(s)^{-1}$ to (1), the lemma follows because $A(s)^{-1} e_{i}(s)=\varepsilon_{i}$ and

$$
u^{*} \Omega(s)=\left(\sum_{i} u^{*} \Omega_{1}^{i} \otimes \varepsilon_{i}, \cdots, \sum_{i} u^{*} \Omega^{i} \otimes \varepsilon_{i}\right) \text {. q.e.d. }
$$

Recall that the map $T_{f}: S \rightarrow G_{3,2}\left(\mathbf{R}^{3}\right)$ is defined by $T_{f}(s)=f_{*} S_{s}$. We need a frame in order to get a local representation of $T_{f}$. Given any point $s \in S$, let $u=\left(e_{1}, e_{2}, e_{3} ; f(s)\right) \in G$ be a zeroth order frame at $s$. Let the 1 -forms $\theta^{i}$ be defined by

$$
d f_{(s)}=f_{*(s)}=\sum_{i} \theta^{i} \otimes e_{i}
$$

Since $d f(s)$ has rank equal to 2 , two of the forms $\theta^{i}$ must be linearly independent. We may as well assume that $\theta^{1}$ and $\theta^{2}$ are independent, in which case $\theta^{3}=x \theta^{1}+y \theta^{2}$ for some $x, y \in \mathbf{R}$. Then, using the representation $G_{3,2}\left(\mathbf{R}^{3}\right)=G x_{G_{o}} G_{3,2}$, we have

$$
T_{f}(s)=\left[u,\left[\begin{array}{ll}
1 & 0 \\
0 & 1 \\
x & y
\end{array}\right]\right]
$$

Any other zeroth order frame at $s$ is given by $\bar{u}=u K$, where $K \in G_{o}$. As

$$
T_{f}(s)=\left[\bar{u} K^{-1},\left[\begin{array}{ll}
1 & 0 \\
0 & 1 \\
x & y
\end{array}\right]\right]=\left[\bar{u}, \rho_{o}\left(K^{-1}\right)\left[\begin{array}{ll}
1 & 0 \\
0 & 1 \\
x & y
\end{array}\right]\right],
$$


it follows that by choosing $K$ appropriately we can simplify the coefficients $x$ and $y$. In fact, the standard action of $G_{o}=S L(3 ; \mathbf{R})$ on $G_{3,2}$ is transitive, which means $K$ can be chosen so that

$$
\rho_{o}\left(K^{-1}\right)\left[\begin{array}{ll}
1 & 0 \\
0 & 1 \\
x & y
\end{array}\right]=\left[\begin{array}{ll}
1 & 0 \\
0 & 1 \\
0 & 0
\end{array}\right] .
$$

Hence $K$ can be chosen so that if $\bar{u}=u K=\left(\bar{e}_{1}, \bar{e}_{2}, \bar{e}_{3} ; f\right)$, then

$$
d f=\bar{\theta}^{1} \otimes \bar{e}_{1}+\bar{\theta}^{2} \otimes \bar{e}_{2}
$$

i.e., $\bar{\theta}^{3}=0$.

Definition. A first order frame at $s \in S$ is any zeroth order frame $u=$ $\left(e_{1}, e_{2}, e_{3} ; f(s)\right) \in G$ such that $d f(s)=\theta^{1} \otimes e_{1}+\theta^{2} \otimes e_{2}$. A first order frame field along $f$ is a zeroth order frame field $u$ along $f$ such that $u(t)$ is a first order frame at $t \in S$ for each $t \in S$.

Although we have defined first order frames in an algebraic way, if we look at the situation geometrically it is clear that the first order frames at $s \in S$ are the zeroth order frames $u=\left(e_{1}, e_{2}, e_{3} ; f(s)\right)$ at $s$ for which $e_{1}$ and $e_{2}$ are tangent to $f(S)$ at $f(s)$.

In order to determine the set of all first order frames at $s \in S$, we need to determine the isotropy subgroup $G_{1}$ of $\rho_{0}$ at the point

$$
\left[\begin{array}{ll}
1 & 0 \\
0 & 1 \\
0 & 0
\end{array}\right] \in G_{3,2} \text {. }
$$

After a brief computation we get that

$$
G_{1}=\left\{K \in G_{o}=S L(3 ; \mathbf{R}): K_{1}^{3}=o=K_{2}^{3}\right\},
$$

where $K_{j}^{i}$ denotes the $i$ th row-jth column entry of the matrix $K$. Then the set of all first order frames at $s$ is

$$
\left\{u K: K \in G_{1}\right\}
$$

where $u$ is any fixed first order frame at $s$.

It can be shown easily, but we omit the details, that first order frame fields along $f$ exist locally, that is, on a neighborhood of any point of $S$. In general there does not exist a continuous first order frame field along $f$ defined on all of $S$. As we are interested here in local questions, we shall often discard portions of $S$, if necessary, so that we may assume that there exist globally defined first order frame fields along $f$.

If we let $L_{1} \subseteq G$ denote the set of all first order frames on $S$, then $L_{1}$ is a submanifold of $G$, and by (2) it is a fibration over $S$ for which each fiber is a right coset of $G_{1}$. The existence of local smooth first order fields along $f$ 
means that the fibration is locally trivial. Thus $L_{1}$ is a principal $G_{1}$-bundle over $S$.

As we shall see, yet another point of view is essential. Any first order frame field $u$ along $f$ is a local cross section of $L_{1} \rightarrow S$, and by Lemma 2

$$
u^{*} \Omega^{3}=0 \text {. }
$$

It follows that $L_{1}$ is an 8-dimensional integral manifold in $G$ of the closed exterior differential system on $G$ :

$$
\Omega^{3}=0, \quad \Omega_{1}^{3} \wedge \Omega^{1}+\Omega_{2}^{3} \wedge \Omega^{2}=0,
$$

where the quadratic equation arises from taking the exterior derivative of $\Omega^{3}=0$ and using the structure equations of $G$.

If $i: L_{1} \rightarrow G$ denotes the inclusion mapping, and if we set $\eta^{i}=i^{*} \Omega^{i}$, $\eta_{j}^{i}=i^{*} \Omega_{j}^{i}$, then $\left(\eta^{1}, \eta^{2}, \eta_{1}^{1}, \eta_{2}^{2}, \eta_{2}^{1}, \eta_{1}^{2}, \eta_{3}^{1}, \eta_{3}^{2}\right)$ is a coframe field on $L_{1}$ and $\eta^{3}=0, \eta_{1}^{3}=p \eta^{1}+q \eta^{2}, \eta_{2}^{3}=q \eta^{1}+r \eta^{2}$ for some smooth functions $p, q$ and $r$ on $L_{1}$.

The following proposition is geometrically obvious, but we include a proof whose idea carries over to less obvious situations.

Proposition 3. Any two surfaces in $\mathbf{R}^{3}$ have first order $\mathbf{G}$-contact at any two points.

Proof. Let $f: S \rightarrow R^{3}$ and $\bar{f}: \bar{S} \rightarrow R^{3}$ be any surfaces, and let $s \in S, \bar{s} \in \bar{S}$. Let $u=\left(e_{1}, e_{2}, e_{3} ; f(s)\right)$ and $\bar{u}=\left(\bar{e}_{1}, \bar{e}_{2}, \bar{e}_{3} ; \bar{f}(\bar{s})\right)$ be first order frames at $s$ and $\bar{s}$, respectively.

For any element $v \in G$ we have $h_{0}(v \cdot \bar{u})=\left(v_{*} e_{1}, v_{*} e_{2}, v_{*} e_{3} ; v \bar{f}(\bar{s})\right)$. Thus $\pi(v \bar{u})=v \cdot \bar{f}(\bar{s})$, so that $v \bar{u}$ is a zeroth order frame at $\bar{s}$ of $v \circ \bar{f}$. Furthermore, the relative components of infinitesimal displacement of $v \circ \bar{f}$ with respect to $h_{0}(v \bar{u})$ are the same as those for $\bar{f}$ with respect to $\bar{u}$. Namely, if $d \bar{f}_{(\mathcal{s})}=\Sigma_{i} \bar{\theta}^{i} \otimes$ $\bar{e}_{i}$, then $d(v \circ \bar{f})_{(s)}=\Sigma_{i} \bar{\theta}^{i} \otimes v_{*} \bar{e}_{i}$.

Let us apply this observation to $v=u \cdot \bar{u}^{-1}$. Then $v \bar{u}=u$ is a first order frame of $v \circ \bar{f}$ at $\bar{s}$ and

$$
T_{v \circ \bar{f}}(\bar{s})=\left[v \bar{u},\left[\begin{array}{ll}
1 & 0 \\
0 & 1 \\
0 & 0
\end{array}\right]\right]=\left[u,\left[\begin{array}{ll}
1 & 0 \\
0 & 1 \\
0 & 0
\end{array}\right]\right]=T_{f}(s) .
$$

Hence $f$ and $v \circ \bar{f}$ have first order contact at $s$ and $\bar{s}$. This proves Proposition 3. q.e.d.

The first order frames can be used to solve the first order $G$-deformation problem. We give an elementary proof of the next proposition. Another proof could be obtained following the methods used for second order deformations.

Proposition 4. Let $f, \bar{f}: S \rightarrow \mathbf{R}^{3}$ be surfaces on which there exist globally defined first order frame fields. Then these surfaces are first order $G$-deformations of each other. 
Proof. Let $u=\left(e_{1}, e_{2}, e_{3} ; f\right): S \rightarrow G$ and $\bar{u}=\left(\bar{e}_{1}, \bar{e}_{2}, \bar{e}_{3} ; \bar{f}\right): S \rightarrow G$ be smooth first order frame fields along $f$ and $\bar{f}$, respectively. Then

$$
f_{*}=\theta^{1} \otimes e_{1}+\theta^{2} \otimes e_{2}, \quad \bar{f}_{*}=\bar{\theta}^{1} \otimes \bar{e}_{1}+\bar{\theta}^{2} \otimes \bar{e}_{2},
$$

where $\theta^{1}, \theta^{2}$ and $\bar{\theta}^{1}, \bar{\theta}^{2}$ are each smooth coframe fields on $S$. There are smooth functions $A_{\beta}^{\alpha}$ on $S$, such that

$$
\bar{\theta}^{\alpha}=\sum_{\beta} A_{\beta}^{\alpha} \theta^{\beta} .
$$

(We are using the index convention: $1 \leqslant \alpha, \beta \leqslant 2$.)

Let $A$ be the $2 \times 2$ matrix of functions $A_{\beta}^{\alpha}$, and let $a=\operatorname{det} A^{-1}$. Define a smooth map $K: S \rightarrow G_{1}$ by

$$
K=\left(\begin{array}{ll}
A & 0 \\
& 0 \\
0 & a
\end{array}\right) .
$$

Then $\bar{u} K$ is also a smooth first order frame field along $\bar{f}$. As $\bar{u} K=$ $\left(\Sigma_{\alpha} A_{1}^{\alpha} \bar{e}_{\alpha}, \Sigma_{\alpha} A_{2}^{\alpha} \bar{e}_{\alpha}, a \bar{e}_{3} ; \bar{f}\right)$, if we set $\bar{u} K=\left(v_{1}, v_{2}, v_{3} ; \bar{f}\right)$, then $v_{\alpha}=\Sigma_{\beta} A_{\alpha}^{\beta} \bar{e}_{\beta}$, and

$$
\bar{f}_{*}=\sum_{\alpha} \varphi^{\alpha} \otimes v_{\alpha}=\sum_{\alpha, \beta} \varphi^{\alpha} \otimes A_{\alpha}^{\beta} \bar{e}_{\beta} .
$$

Comparing (5) with the second equation in (3), it follows that

$$
\bar{\theta}^{\alpha}=\sum_{\beta} A_{\beta}^{\alpha} \varphi^{\beta} .
$$

Comparing (6) with (4) gives that $\varphi^{\beta}=\theta^{\beta}$.

The point of this brief digression is that we may assume the first order frame field $\bar{u}$ along $\bar{f}$ to be chosen such that

$$
\bar{\theta}^{\alpha}=\theta^{\alpha} \text {. }
$$

Define a smooth map $v: S \rightarrow G$ by $v(s)=u(s) \bar{u}(s)^{-1}$. Given any $s \in S$, it is clear that $v(s)_{*} \bar{e}_{\alpha}(s)=e_{\alpha}(s)$. Thus using (7) we have

$$
(v(s) \circ \bar{f})_{*}(s)=v(s)_{*}\left(\sum_{\alpha} \theta^{\alpha} \otimes \bar{e}_{\alpha}(s)\right)=\sum_{\alpha} \theta^{\alpha} \otimes e_{\alpha}(s)=f_{*}(s),
$$

which means that $v(s) \circ \bar{f}$ agrees to at least first order with $f$ at $s$. q.e.d.

We shall need second order frames and the notion of the second order types in $\$ \S 9$ and 10. Rather than just refer to their construction in [7, pp. 118-119], we shall 'repeat the construction, but emphasize the pointwise nature of their definition.

Lemma 3. Let $\sigma_{o}$ denote the 2-plane at the origin of $\mathbf{R}^{3}$ spanned by the two standard basis vectors $\varepsilon_{1}$ and $\varepsilon_{2}$. Then the map $v \rightarrow v_{*} \sigma_{o}$, for $v \in G$, induces $a$ bundle isomorphism $G / G_{1} \cong G_{3,2}\left(\mathbf{R}^{3}\right)$. 
Proof. Any tangent 2-plane to $\mathrm{R}^{3}$ is given by $v_{*} \sigma_{o}$ for some $v \in G$; i.e., the map is surjective. The proof is concluded by observing that $v_{*} \sigma_{o}=\sigma_{o}$ if and only if $v(o)=0$ and

$$
\rho_{0}(v)\left[\begin{array}{ll}
1 & 0 \\
0 & 1 \\
0 & 0
\end{array}\right]=\left[\begin{array}{ll}
1 & 0 \\
0 & 1 \\
0 & 0
\end{array}\right]
$$

i.e., $v \in G_{1}$. q.e.d.

In terms of our earlier notation, the isomorphism

$$
G / G_{1} \cong G_{3,2}\left(\mathbf{R}^{3}\right) \cong G x_{G_{o}} G_{3,2}
$$

is given by

$$
u \cdot G \rightarrow\left[u,\left[\begin{array}{ll}
1 & 0 \\
0 & 1 \\
0 & 0
\end{array}\right]\right]
$$

Using this identification we have

$$
T_{f}: S \rightarrow G / G_{1} .
$$

Let $\pi_{1}: G \rightarrow G / G_{1}$ denote the natural projection. A zeroth order frame for $T_{f}$ at $s \in S$ is an element $u \in G$ for which $\pi_{1}(u)=T_{f}(s)$. Then $u \in G$ is a zeroth order frame for $T_{f}$ at $s$ if and only if

$$
T_{f}(s)=\pi_{1}(u)=\left[u,\left[\begin{array}{ll}
1 & 0 \\
0 & 1 \\
0 & 0
\end{array}\right]\right],
$$

which occurs if and only if $u$ is a first order frame for $f$ at $s$.

Next we determine the relative components of infinitesimal displacement of $T_{f}$ with respect to a zeroth order frame. The second order frames for $f$ at a point $s \in S$ will be defined to be the first order frames for $T_{f}$ at $s$.

The construction of first order frames for $T_{f}$ begins with a choice of subspace $\mathfrak{m}_{1}$ of $g_{o}$ complementary to the Lie algebra $g_{1}$ of $G_{1}$. Recall that $\mathfrak{g}_{o}=\mathfrak{s} \mathfrak{I}(3 ; \mathbf{R})$ and that

$$
\mathfrak{g}_{1}=\left\{X \in \mathfrak{g}_{o}: X_{1}^{3}=0=X_{2}^{3}\right\} .
$$

For $\mathrm{m}_{1}$ we take

$$
\mathfrak{m}_{1}=\left\{\left(\begin{array}{lll}
0 & 0 & 0 \\
0 & 0 & 0 \\
r & t & 0
\end{array}\right) \in \mathfrak{g}_{o}:, t \in R\right\} .
$$

Then $\mathfrak{g}=\mathfrak{g}_{1} \oplus \mathfrak{m}_{o} \oplus \mathfrak{m}_{1}$, (vector space direct sum), and $\pi_{1 *}$ maps $\mathfrak{m}_{o} \oplus \mathfrak{m}_{1}$ isomorphically onto the tangent space of $G / G_{1}$ at the identity coset. 
As a reference frame at the identity coset of $G / G_{1}$ we take $u_{1}=$ $\pi_{1 *}\left(\varepsilon_{1}, \varepsilon_{2}, \varepsilon_{3}, \varepsilon_{4}, \varepsilon_{5}\right)$, where $\varepsilon_{1}, \varepsilon_{2}, \varepsilon_{3}$ continues to be the standard basis of $\mathfrak{m}_{0} \cong \mathbf{R}^{3}$, and

$$
\varepsilon_{4}=\left(\begin{array}{lll}
0 & 0 & 0 \\
0 & 0 & 0 \\
1 & 0 & 0
\end{array}\right), \quad \varepsilon_{5}=\left(\begin{array}{lll}
0 & 0 & 0 \\
0 & 0 & 0 \\
0 & 1 & 0
\end{array}\right)
$$

is a basis of $\mathfrak{m}_{1}$. Then we get a bundle monomorphism $h_{1}: G \rightarrow L\left(G / G_{1}\right)$ given by $h_{1}(u)=u_{*} u_{1}$. The associated homomorphism between the structure groups $\rho_{1}: G_{1} \rightarrow G L(5 ; \mathbf{R})$ is the adjoint representation of $G_{1}$ on $\mathfrak{g} / \mathfrak{g}_{1} \simeq \mathfrak{m}_{o}$ $\bigoplus \mathrm{m}_{1}$, with respect to the basis $\varepsilon_{1}, \cdots, \varepsilon_{5}$.

As $G_{1} \subset G_{o}$, there is a well-defined smooth projection $\psi_{1}: G / G_{1} \rightarrow G / G_{o}$ given by $\pi_{1}(v) \rightarrow \pi(v)$, for any $v \in G$. Then

$$
\psi_{1 *} \pi_{1 *}\left(\varepsilon_{1}, \cdots, \varepsilon_{5}\right)=\pi_{*}\left(\varepsilon_{1}, \varepsilon_{2}, \varepsilon_{3}\right), \quad \psi_{1} \circ T_{f}=f
$$

Notice the equivariance property of $\psi_{1}$, namely that for $v, w \in G, \psi_{1}(v$. $\left.\pi_{1}(w)\right)=v \cdot \pi(w)$. Thus for any $v \in G$, if $h_{o}(v)=\left(e_{1}, e_{2}, e_{3} ; \pi(v)\right)$ and $h_{1}(v)$ $=\left(\tilde{e}_{1}, \tilde{e}_{2}, \tilde{e}_{3}, e_{4}, e_{5} ; \pi_{1}(v)\right)$, then

$$
\psi_{1 *} h_{1}(v)=\left(\psi_{1 *} \tilde{e}_{1}, \cdots, \psi_{1 *} e_{5} ; \psi_{1} \circ \pi_{1}(v)\right)=\left(e_{1}, e_{2}, e_{3} ; \pi(v)\right)=h_{o}(v)
$$

As a result of (8) we shall identify $\tilde{e}_{i}$ with $e_{i}, i=1,2,3$.

Lemma 4. Let $u$ be a first order frame for $f$ at $s \in S$, and set $h_{o}(u)=$ $\left(e_{1}, e_{2}, e_{3} ; f(s)\right)$ and $h_{1}(u)=\left(e_{1}, \cdots, e_{5} ; T_{f}(s)\right)$. Then

$$
\left(T_{f}\right)_{*(s)}=\sum_{\alpha} \theta^{\alpha} \otimes e_{\alpha}+\omega_{1}^{3} \otimes e_{4}+\omega_{2}^{3} \otimes e_{5},
$$

where $f_{*(s)}=\Sigma_{\alpha} \theta^{\alpha} \otimes e_{\alpha}$ and $\omega_{1}^{3}, \omega_{2}^{3}$ are linear functionals on the tangent space $S_{s}$.

If $u$ is a first order frame field along $f$, and thus is a zeroth order frame field along $T_{f}$, then the relative components of infinitesimal displacement of $T_{f}$ with respect to $u$, as defined in (9) at any point $s \in S$, are

$$
\theta^{\alpha}=u^{*} \Omega^{\alpha}, \quad u^{*} \Omega^{3}=0, \quad \omega_{1}^{3}=u^{*} \Omega_{1}^{3}, \quad \omega_{2}^{3}=u^{*} \Omega_{2}^{3} .
$$

Furthermore, the structure equation

$$
\omega_{1}^{3} \wedge \theta^{1}+\omega_{2}^{3} \wedge \theta^{2}=0
$$

is satisfied on $S$.

Proof. (9) is satisfied for some linear functionals $\theta^{\alpha}, \omega_{1}^{3}, \omega_{2}^{3} \in S_{s}^{*}$. As $f=\psi_{1} \circ T_{f}$, by (8) we have $f_{*(s)}=\psi_{1 *}\left(\Sigma_{\alpha} \theta^{\alpha} \otimes e_{\alpha}+\omega_{1}^{3} \otimes e_{4}+\omega_{2}^{3} \otimes e_{5}\right)=$ $\Sigma_{\alpha} \theta^{\alpha} \otimes e_{\alpha}$. 
The proof of the latter half of the lemma is the same as the proof of Lemma 2. (11) follows from applying $u^{*}$ to the following structure equation of $G$ :

$$
d \Omega^{3}=-\sum_{i} \Omega_{i}^{3} \wedge \Omega^{i} . \text { q.e.d. }
$$

Continue using the notation of Lemma $4 ; u$ is a first order frame for $f$ at $s \in S$. From (11)

$$
\omega_{1}^{3}=x \theta^{1}+y \theta^{2}, \quad \omega_{2}^{3}=y \theta^{1}+z \theta^{2}
$$

for some $x, y, z \in \mathbf{R}$. Let $X=\left(\begin{array}{ll}x & y \\ y & z\end{array}\right)$, and let $\varsigma_{2}$ denote the vector space of all real $2 \times 2$ symmetric matrices.

Now $T_{f}^{2}: S \rightarrow G_{5,2}\left(M^{1}\right)$, and using $h_{1}$ we see that the latter space is given by

$$
G_{5,2}\left(M^{1}\right)=G x_{G_{1}} G_{5,2},
$$

where we recall that $\rho_{1}: G_{1} \rightarrow G L(5 ; \mathbf{R})$ is the adjoint representation of $G_{1}$ on $\mathrm{g} / \mathrm{g}_{1}$ with respect to the basis $\varepsilon_{1}, \cdots, \varepsilon_{5}$. Then by (9) we have

$$
T_{f}^{2}(s)=\left[u,\left[\begin{array}{l}
I \\
0 \\
X
\end{array}\right]\right]
$$

where $I=\left(\begin{array}{ll}1 & 9 \\ 0 & 1\end{array}\right)$, and $X$ is given above.

Any other first order frame for $f$ at $s$ is given by $\bar{u}=u K$ for some $K \in G_{1}$. If $h_{1}(\bar{u})=\left(\bar{e}, \cdots, \bar{e}_{5} ; \pi_{1}(\bar{u})\right)$, and we denote the relative components of $T_{f}$ with respect to $h_{1}(\bar{u})$ by $\bar{\theta}^{\alpha}, \bar{\omega}_{1}^{3}, \bar{\omega}_{2}^{3}$, and denote the coefficients of the latter by $X=\left(\bar{x} \bar{y} \frac{\bar{y}}{\bar{z}}\right)$, then

$$
\left[\begin{array}{l}
I \\
0 \\
\bar{X}
\end{array}\right]=\rho_{1}\left(K^{-1}\right)\left[\begin{array}{l}
I \\
0 \\
X
\end{array}\right]
$$

Lemma 5. Let $K \in G_{1}$, so that $K=\left(\begin{array}{cc}A & \xi \\ 0 & a\end{array}\right)$, for some $A \in G L(2 ; \mathbf{R})$, some $\xi \in \mathbf{R}^{2}$, and where $a^{-1}=\operatorname{det} A$. Then

$$
\operatorname{Ad}\left(K^{-1}\right)\left(\left[\begin{array}{ll}
0 & 0 \\
& 0 \\
\omega_{1}^{3} \omega_{2}^{3} & 0
\end{array}\right),\left(\begin{array}{l}
\theta^{1} \\
\theta^{2} \\
0
\end{array}\right)\right]=\left(\left[\begin{array}{ll}
0 & 0 \\
a^{-1}\left(\omega_{1}^{3} \omega_{2}^{3}\right) A & 0
\end{array}\right],\left(\begin{array}{c}
\theta^{1} \\
\theta^{2}
\end{array}\right)\right], \text { modulo } g_{1}
$$

Consequently in (12),

$$
\bar{X}=a^{-1 t} A X A
$$


Proof. An elementary computation.

The action of $G_{1}$ on $\delta_{2}$ defined by (13) has exactly four distinct orbits. A representative from each orbit is chosen as follows:

Type IIa: Orbit of $0=X_{a}$.

Type IIb: Orbit of $\left(\begin{array}{ll}1 & 9 \\ 0 & 0\end{array}\right)=X_{b}$.

Type IIc: Orbit of $\left(\begin{array}{ll}1 & 0 \\ 0 & 1\end{array}\right)=X_{c}$.

Type IId: Orbit of $\left(\begin{array}{cc}1 & 0 \\ 0 & -1\end{array}\right)=X_{d}$.

If $X$ lies in the orbit of Type IIa (respectively, Type IIb, or Type IIc, or Type IId), then the point $s \in S$ is called a planar (respectively, parabolic, or elliptic, or hyperbolic) point. The surface $f: S \rightarrow R^{3}$ is said to be of constant second order type if all of its points are of the same second order type. If $f$ is of constant second order type, we shall call it planar (respectively parabolic, elliptic or hyperbolic), if all of its points are planar (respectively parabolic, elliptic or hyperbolic).

Definition. A second order frame for $f$ at $s \in S$ is any first order frame $u$ at $s$ for which

$$
T_{f}^{2}(s)=\left[u,\left[\begin{array}{c}
I \\
0 \\
X_{D}
\end{array}\right]\right]
$$

where $D$ is one of $a, b, c$, or $d$.

It can be shown that if $f$ is of constant second order type, then locally there exist smooth second order frame fields along $f$. Let $L_{2}$ denote the set of all second order frames on $f$. If $f$ has constant second order type IID, then $L_{2} \rightarrow S$ is a principal $G_{2}$-bundle over $S$, where $G_{2}$ is the isotropy subgroup of $G_{1}$ at $X_{D}, D=a, b, c, d$ :

$$
G_{2}=\left\{K \in G_{1}: \rho_{1}(K)\left[\begin{array}{c}
I \\
0 \\
X_{D}
\end{array}\right]=\left[\begin{array}{c}
I \\
0 \\
X_{D}
\end{array}\right]\right\}
$$

Proposition 5. Smooth surfaces $f: S \rightarrow R^{3}$ and $\bar{f}: \bar{S} \rightarrow R^{3}$ have second order $G$-contact at $s \in S$ and $\bar{s} \in \bar{S}$ if and only if $s$ and $\bar{s}$ are of the same second order type.

Proof. The proof is similar to that of Proposition 3.

\section{The differential systems of equiaffine deformations}

We shall use the results of Example 1 in $\$ 4$ for the case when $G$ is the equiaffine group. Adopt the index conventions

$$
1 \leqslant i, j \leqslant 3, \quad 1 \leqslant \alpha, \beta \leqslant 2,
$$


and use the Einstein summation convention on repeated indices. Then equations (4.6) from Example 1 are

$$
d x^{i}=-\Omega^{i}-x^{j} \Omega_{j}^{i}, \quad\left(\text { on } G \times \mathbf{R}^{3}\right) .
$$

Suppose that $f, \bar{f}: S \rightarrow M$ are surfaces which are $k$ th order equiaffine deformations of each other. Let $v: S \rightarrow G$ be such that $v(s) \circ \bar{f}$ agrees through order $k$ with $f$ at $s$, for every $s \in S$.

Let $u: S \rightarrow L_{k}$ be a $k$ th order frame field along $f$. Then $\bar{u}: S \rightarrow G$ defined by $\bar{u}(s)=v(s)^{-1} \cdot u(s)$ is a $k$ th order frame field along $\bar{f}$. For each $(s, t) \in S$ $\times S$, the coordinates $\left(x^{i}\right)$ of $f(t)$ with respect to $u(s)$ satisfy

$$
f(t)=f(s)+x^{i}(s, t) e_{i}(s),
$$

where, as in Example 1, $u(s)=\left(e_{1}(s), e_{2}(s), e_{3}(s) ; f(s)\right)$. The coordinates $\left(\bar{x}^{i}\right)$ of $\bar{f}(t)$ with respect to $\bar{u}(s)$ are the same as the coordinates of $v(s) \circ \bar{f}(t)$ with respect to $u(s)$, so that

$$
v(s) \circ \bar{f}(t)=f(s)+\bar{x}^{i}(s, t) e_{i}(s) .
$$

Put $\omega^{i}=u^{*} \Omega^{i}, \omega_{j}^{i}=u^{*} \Omega_{j}^{i}$, and similarly define $\bar{\omega}^{i}$ and $\bar{\omega}_{j}^{i}$ with respect to $\bar{u}$. If $u$ is at least a first order frame field, then $\omega^{3}=0$ and $\left\{\omega^{1}, \omega^{2}\right\}$ is a coframe field on $S$. Consequently, for each $s \in S$, the functions $x^{\alpha}(s, t)$ define local coordinates in $S$ centered at $s$. Then

$$
x^{3}=a_{\alpha} x^{\alpha}+\frac{1}{2} b_{\alpha \beta} x^{\alpha} x^{\beta}+\cdots \cdot
$$

Where the coefficients $a_{\alpha}, b_{\alpha \beta}=b_{\beta \alpha}, \cdots$ are functions of $s$.

Use the map ( $u \times$ identity): $S \times \mathbf{R}^{3} \rightarrow G \times \mathbf{R}^{3}$ to pull (14) back to $S \times$ $\mathbf{R}^{3}$ :

$$
d x^{i}=-\omega^{i}-x^{j} \omega_{j}^{i}
$$

Differentiate (15) with respect to $s$, keeping $t$ fixed, so that equations (16) can be used. Thus we obtain

$$
\omega_{i}^{3} x^{i}=d x^{3}=a_{\alpha} d x^{\alpha}+x^{\alpha} d a_{\alpha}+\cdots .
$$

At $s=t$ we have $x^{i}=0$ and $d x^{1}, d x^{2}$ linearly independent. Hence $a_{\alpha}=0$, and the expansion (15) begins with the second order term.

Now $v(s) \circ \bar{f}$ and $f$ agree to order $k$ at $s$; it means that $\bar{x}^{i}-x^{i}=0$ modulo terms of order greater than $k$ in $x^{1}, x^{2}$.

Case $k=1$. Differentiate $\bar{x}^{i}-x^{i}$ with respect to $s$, keeping $t$ fixed. Then Using (16) we obtain

$$
d \bar{x}^{i}=d x^{i} \text { modulo terms of order } \geqslant 1 \text { in } x^{\alpha} \text {. }
$$

$$
\bar{\omega}^{i}+\bar{x}^{j} \bar{\omega}_{j}^{i}=\omega^{i}+x^{j} \omega_{j}^{i} \quad(\bmod \text { order } \geqslant 1) .
$$


Hence putting $s=t$ gives

$$
\bar{\omega}^{i}=\omega^{i} \quad \text { on } S .
$$

These are the exterior differential equations of a first order equiaffine deformation.

Case $k=2$.

Now $d \bar{x}^{i}=d x^{i}\left(\bmod\right.$ order $\geqslant 2$ in $\left.s^{\alpha}\right)$. Using (16) we have

$$
\bar{\omega}^{i}+\bar{x}^{\alpha} \bar{\omega}_{\alpha}^{i}=\omega^{i}+x^{\alpha} \omega_{\alpha}^{i} \quad\left(\bmod \text { order } \geqslant 2 \text { in } x^{\alpha}\right) .
$$

But $\bar{x}^{\alpha}=x^{\alpha}$ through second order, and $\bar{\omega}^{i}=\omega^{i}$ as in the case $k=1$. Thus $x^{\alpha}\left(\bar{\omega}_{\alpha}^{i}-\omega_{\alpha}^{i}\right)=0$ modulo terms of order $\geqslant 2$ in $x^{\alpha}$. By a standard argument

$$
\bar{\omega}_{\alpha}^{i}=\omega_{\alpha}^{i} \quad \text { on } S .
$$

The differential equations of a second order equiaffine deformation are the equations

$$
\bar{\omega}^{i}=\omega^{i}, \quad \bar{\omega}_{\alpha}^{i}=\omega_{\alpha}^{i} \text { on } S
$$

Case $k=3$. Now

$$
d \bar{x}^{i}=d x^{i} \quad\left(\bmod \text { order } \geqslant 3 \text { in } x^{\alpha}\right) .
$$

Using (15) and (16) we obtain

$$
\begin{aligned}
\bar{\omega}^{i}+\bar{x}^{\alpha} \bar{\omega}_{\alpha}^{i}+\left(\frac{1}{2} \bar{b}_{\alpha \beta} \bar{x}^{\alpha} \bar{x}^{\beta}\right) \bar{\omega}_{3}^{i}=\omega^{i}+x^{\alpha} \omega_{\alpha}^{i}+\left(\frac{1}{2} b_{\alpha \beta} x^{\alpha} x^{\beta}\right) \omega_{3}^{i}, \\
\text { modulo terms of order }>3 \text { in } x^{\alpha} .
\end{aligned}
$$

Since $\bar{x}^{i}=x^{i}$ through third order, and $\bar{\omega}^{i}=\omega^{i}, \bar{\omega}_{\alpha}^{i}=\omega_{\alpha}^{i}$ from Cases $k=1,2$, we have $\bar{b}_{\alpha \beta}=b_{\alpha \beta}$ and

$$
b_{\alpha \beta} x^{\alpha} x^{\beta}\left(\bar{\omega}_{3}^{i}-\omega_{3}^{i}\right)=0 \quad(\bmod \text { order } \geqslant 3) .
$$

Hence either

$$
\bar{\omega}_{3}^{i}=\omega_{3}^{i} \quad \text { on } S,
$$

or $b_{\alpha \beta}$ is identically zero on $S$ for all $\alpha, \beta$.

Generically the former case occurs, so that (17), (18), and (20) are the differential equations of a third order equiaffine deformation.

Proposition. Any $C^{\infty}$ surface in $\mathbf{R}^{3}$ is rigid to local third order equiaffine deformations.

Proof. Surfaces for which the $b_{\alpha \beta}$ are identically 0 are planar surfaces, and any planar surface is an open submanifold of a plane (cf. [7, p. 120]). Since a third order deformation of a planar surface is also planar, they are congruent.

For a nonplanar surface, the equations are (17), (18), and (20) above, which say that $u^{*} \Omega=\bar{u}^{*} \Omega$. It follows that $f$ and $\bar{f}$ are $G$-congruent. 


\section{Existence of deformations}

We have seen already that any two surfaces are locally first order equiaffine deformations of each other, and that every surface is rigid to third order equiaffine deformations. We turn now to the questions raised in $\$ 7$ for the case of second order equiaffine deformations of surfaces.

The Cartan-Kähler theorem will be used many times in this and the next sections. For terminology and precise formulation of this theorem we shall follow [4], especially $\$ \$ 93$ and 94 on pp. 98-100.

The answer to question (1) is a qualified no; that is, a generic surface is rigid to second order deformations. We proceed directly to question (2) to see which surfaces are deformable. Since there are four second order types of surfaces, we can unify the discussion somewhat by setting up our first system on $L_{1} \times G$, rather than on $L_{2} \times G$.

Let $f: S \rightarrow \mathbf{R}^{3}$ be a real analytic surface, and let $\Omega=\left(\left(\Omega_{j}^{i}\right),\left(\Omega^{i}\right)\right)$ denote the Maurer-Cartan form of $G$. The set $L_{1}$ of all first order frames on $f$ is a real analytic submanifold of $G$. In fact, $L_{1}$ is an 8-dimensional integral manifold of the closed exterior differential system

$$
\Omega^{3}=0, \quad \Omega_{1}^{3} \wedge \Omega^{1}+\Omega_{2}^{3} \wedge \Omega^{2}=0
$$

on $G$.

Let $i: L_{1} \rightarrow G$ denote the inclusion mapping, and set

$$
\omega^{i}=i^{*} \Omega^{i}, \quad \omega_{j}^{i}=i^{*} \Omega_{j}^{i}, \quad 1 \leqslant i, j \leqslant 3 .
$$

Then $\omega^{1}, \omega^{2}, \omega_{1}^{1}, \omega_{2}^{2}, \omega_{2}^{1}, \omega_{3}^{1}, \omega_{1}^{2}, \omega_{3}^{2}$ form a coframe field on $L_{1}$, and

$$
\omega^{3}=0, \quad \omega_{1}^{3}=p \omega^{1}+q \omega^{2}, \quad \omega_{2}^{3}=q \omega^{1}+r \omega^{2},
$$

for some real analytic functions $p, q$ and $r$ on $L_{1}$.

Consider the closed exterior differential system $\Sigma$ on $L_{1} \times G$ with specified independent variables $\omega^{1}, \omega^{2}$, (cf. (19)):

$$
\begin{aligned}
& \omega^{1}=\Omega^{1}, \quad \omega^{2}=\Omega^{2}, \quad \Omega^{3}=0, \quad \omega_{1}^{3}=\Omega_{1}^{3}, \quad \omega_{2}^{3}=\Omega_{2}^{3}, \\
& \omega_{2}^{1}=\Omega_{2}^{1}, \quad \omega_{1}^{1}=\Omega_{1}^{1}, \quad \omega_{2}^{2}=\Omega_{2}^{2}, \quad \omega_{1}^{2}=\Omega_{1}^{2} ; \\
& \left(\omega_{3}^{1}-\Omega_{3}^{1}\right) \wedge \omega_{1}^{3}=0, \quad\left(\omega_{3}^{1}-\Omega_{3}^{1}\right) \wedge \omega_{2}^{3}=0, \\
& \left(\omega_{3}^{2}-\Omega_{3}^{2}\right) \wedge \omega_{1}^{3}=0, \quad\left(\omega_{3}^{2}-\Omega_{3}^{2}\right) \wedge \omega_{2}^{3}=0 .
\end{aligned}
$$

The nature of this exterior differential system depends on the linear dependence between $\omega_{1}^{3}$ and $\omega_{2}^{3}$, which in turn depends on the second order type of the surface $f$.

By the Remark following Proposition 2, if $\bar{f}$ is a second order $G$-deformation of $f$, and $f$ has constant second order type, then $\bar{f}$ has the same constant 
second order type. For surfaces of constant second order type we have the following results.

Proposition 8. Any planar, elliptic, or hyperbolic real analytic surface in $R^{3}$ is rigid to second order $G$-deformations. Any real analytic parabolic surface in $R^{3}$ has local nontrivial second order $G$-deformations.

Proof. The surface $f$ is planar iff $p, q$ and $r$ are identically zero. In this case the system $\Sigma$ is completely integrable. Any two-dimensional solution is given by a real analytic map $(u, \bar{u}): S \rightarrow L_{1} \times G$, and the surfaces $f=\pi \circ u$ and $\bar{f}=\pi \circ \bar{u}$ are $G$-congruent. They are each open submanifolds of a plane. (Cf. [7, p. 120].)

The surface $f$ is elliptic iff $p r-q^{2}>0$, and it is hyperbolic iff $p r-q^{2}<0$. In either case the four quadratic equations (35) are linearly independent, while the rank of the polar matrix is 2 . The system $\Sigma$ is not in involution.

A prolongation of the system is achieved by adding to (34) the linear equation's

$$
\bar{\Omega}_{3}^{1}-\Omega_{3}^{1}=a \Omega^{1}+b \Omega^{2}, \quad \bar{\Omega}_{3}^{2}-\Omega_{3}^{2}=c \Omega^{1}+e \Omega^{2},
$$

where $a, b, c, e$ are new variables. Putting equation's (36) into the quadratic equations (35), it follows that $a, b, c$, and $e$ all must be zero.

Thus the prolongation is completely integrable. Any solution is given by $(u, \bar{u}): S \rightarrow L_{1} \times G$ and satisfies $u^{*} i^{*} \Omega=\bar{u}^{*} \Omega$. Hence $f=\pi \circ u$ and $\bar{f}=$ $\pi \circ \bar{u}$ are $G$-congruent by [7, Theorem 1, p. 30].

The surface $f$ is parabolic iff the matrix $\left(\begin{array}{ll}p & q \\ q & r\end{array}\right)$ has rank one at every point of $L_{1}$. In this case there are only two independent quadratic equations in (35), and the system $\Sigma$ is in involution. The general solution depends on two arbitrary functions of one variable.

\section{Deformations of parabolic surfaces}

We conclude with the problem of determining the second order $G$-deformations of a given parabolic surface $f: S \rightarrow R^{3}$. For the details on higher order frames and types of parabolic surfaces we refer to [7, pp. 120-139].

Let $L_{2} \subseteq G$ be the set of second order frames of $f$. It is a principal $G_{2}$-bundle over $S$, where (cf. §7)

$$
G_{2}=\left\{\left[\begin{array}{lll}
a & 0 & c \\
b & a^{-3} & t \\
0 & 0 & a^{2}
\end{array}\right]: a, b, c, t \in \mathbf{R}, a \neq 0\right\}
$$


Also, $L_{2}$ is a 6-dimensional integral manifold of the closed exterior differential system on $G$ :

$$
\begin{gathered}
\Omega^{3}=0, \quad \Omega_{1}^{3}=\Omega^{1}, \quad \Omega_{2}^{3}=0, \quad \Omega_{2}^{1} \wedge \Omega^{1}=0, \\
\Omega^{1} \wedge\left(3 \Omega_{1}^{1}+\Omega_{2}^{2}\right)=\Omega_{2}^{1} \wedge \Omega^{2} .
\end{gathered}
$$

Let $i: L_{2} \rightarrow G$ be the inclusion mapping, and set $\omega^{i}=i^{*} \Omega^{i}, \omega_{j}^{i}=i^{*} \Omega_{j}^{i}$. Then $\left(\omega^{1}, \omega^{2}, \omega_{1}^{1}, \omega_{3}^{1}, \omega_{1}^{2}, \omega_{3}^{2}\right)$ is a coframe field on $L_{2}$, and $\omega^{3}=0, \omega_{1}^{3}=\omega^{1}$, $\omega_{2}^{3}=0, \omega_{2}^{1}=q \omega^{1}, 3 \omega_{1}^{1}+\omega_{2}^{2}=p \omega^{1}+q \omega^{2}$, where $p, q$ are real analytic functions on $L_{2}$.

There are two third order types, according to whether $\omega_{2}^{1}=0$, (Type IIb IIIa), or $\omega_{2}^{1} \neq 0$, (Type IIb IIIb). We have observed already that second order deformations preserve second order type. But from (19) (namely, $\bar{\omega}_{2}^{1}=\omega_{2}^{1}$ ), it follows that second order deformations of a parabolic surface preserve third order type. To see this one must observe that if $\bar{f}: S \rightarrow \mathbf{R}^{3}$ is a second order deformation of $f: S \rightarrow \mathbf{R}^{3}$, and the deformation is achieved by $v$ : $S \rightarrow G$, then starting with a second order frame field $u: S \rightarrow G$ along $f$ we get a second order frame field $\bar{u}: S \rightarrow G$ along $\bar{f}$. by setting $\bar{u}(s)=v(s)^{-1} \cdot u(s)$.

Suppose that $f$ is of Type IIb IIIa, and let $L_{3} \subseteq L_{2} \subseteq G$ denote its set of third order frames. $L_{3}$ is a principal $G_{3}$-bundle over $S$, where

$$
G_{3}=\left\{\left(\begin{array}{lll}
a & 0 & 0 \\
b & a^{-3} & t \\
0 & 0 & a^{2}
\end{array}\right]: a, b, t \in \mathbf{R}, a \neq 0\right\} ;
$$

and $L_{3}$ is a 5-dimensional integral manifold of the closed exterior differential system on $G$ :

(38) $\Omega^{3}=0, \quad \Omega_{1}^{3}=\Omega^{1}, \quad \Omega_{2}^{3}=0, \quad \Omega_{2}^{1}=0, \quad 3 \Omega_{1}^{1}+\Omega_{2}^{2}=0, \quad \Omega_{3}^{1} \wedge \Omega^{1}=0$.

If we now let $i: L_{3} \rightarrow G$ denote this inclusion, and let $\omega^{i}=i^{*} \Omega^{i}, \omega_{j}^{i}=i^{*} \Omega_{j}^{i}$, then $\left(\omega^{1}, \omega^{2}, \omega_{1}^{1}, \omega_{1}^{2}, \omega_{3}^{2}\right)$ is a coframe field on $L_{3}$, and

$$
\omega^{3}=0, \quad \omega_{1}^{3}=\omega^{1}, \quad \omega_{2}^{3}=0, \quad \omega_{2}^{1}=0, \quad \omega_{2}^{2}=-3 \omega_{1}^{1}, \quad \omega_{3}^{1}=r \bar{\omega}^{1}
$$

for some function $r$ on $L_{3}$.

With this preparation we can state and prove

Proposition 9. Any Type IIb IIIa surface $\bar{f}: \bar{S} \rightarrow \mathbf{R}^{3}$ is a second order $G$-deformation of $f$.

Proof. Let any quantity defined above for $f$ be defined for $\bar{f}$, and denote it with the same letter but with a bar over it.

From (19), (39) and (39), it suffices to find a two-dimensional solution to the closed exterior differential system on $L_{3} \times \bar{L}_{3}$ with specified independent variables $\omega^{1}, \omega^{2}$ :

$$
\bar{\omega}^{1}=\omega^{1}, \quad \bar{\omega}^{2}=\omega^{2}, \quad \bar{\omega}_{1}^{1}=\omega_{1}^{1}, \quad \bar{\omega}_{1}^{2}=\omega_{1}^{2}, \quad\left(\bar{\omega}_{3}^{2}-\omega_{3}^{2}\right) \wedge \omega^{1}=0 .
$$


This system is in involution, and the general solution depends on 1 arbitrary function of 1 variable. Any 2-dimensional solution can be given by a map

$$
(u, \bar{u}): S \rightarrow L_{3} \times \bar{L}_{3} \text {. }
$$

Then $u$ and $\bar{u}$ are third order frame fields along $f$ and $\pi \circ \bar{u}$, respectively, and

$$
\begin{aligned}
& \bar{u}^{*} \bar{\omega}^{1}=u^{*} \omega^{1}, \quad \bar{u}^{*} \bar{\omega}^{2}=u^{*} \omega^{2}, \quad \bar{u}^{*} \bar{\omega}_{1}^{3}=\bar{u}^{*} \bar{\omega}_{1}^{3}, \\
& \bar{u}^{*} \bar{\omega}_{2}^{3}=u^{*} \omega_{2}^{3}, \quad \bar{u}^{*} \bar{\omega}_{1}^{1}=u^{*} \omega_{1}^{1}, \quad \bar{u}^{*} \bar{\omega}_{2}^{2}=u^{*} \omega_{2}^{2}, \\
& \bar{u}^{*} \bar{\omega}_{2}^{1}=u^{*} \omega_{2}^{1}, \quad \bar{u}^{*} \bar{\omega}_{1}^{2}=u^{*} \omega_{1}^{2} .
\end{aligned}
$$

By Proposition 6, $f$ and $\pi \circ \bar{u}$ are second order $G$-deformations of each other. Finally, $\pi \circ \bar{u}(S)=\pi\left(\bar{L}_{3}\right)=\bar{f}(\bar{S})$, so that $\pi \circ \bar{u}: S \rightarrow \mathbf{R}^{3}$ is just a reparametrization of $\bar{f}$.

Remark. Type IIb IIIa surfaces $f: S \rightarrow \mathbf{R}^{3}$ are cylinders. To see this, let $u$ : $S \rightarrow L_{3}$ be a third order frame field along $f$, and write $u=\left(e_{1}, e_{2}, e_{3} ; f\right)$.

If $u^{*} \Omega^{i}=\theta^{i}, i=1,2, u^{*} \Omega_{j}^{i}=\theta_{j}^{i}$, then we have

$$
d e_{2}=\theta_{2}^{2} \otimes e_{2}
$$

as $\theta_{2}^{1}=0=\theta_{2}^{3}$. It follows that the tangent vector field $e_{2}$ along $f$ has constant direction, and thus $f$ is a cylinder generated by lines parallel to $e_{2}$.

Consider now a Type IIb IIIb surface $f: S \rightarrow \mathbf{R}^{3}$, and let $L_{3} \subseteq G$ denote its set of third order frames. $L_{3}$ is a principal $G_{3}$-bundle over $S$ where now

$$
G=\left\{\left(\begin{array}{ccc}
1 & 0 & b \\
b & 1 & t \\
0 & 0 & 1
\end{array}\right): b, t \in \mathbf{R}\right\}
$$

It is also a 4-dimensional integral manifold of the closed exterior differential system on $G$ :

$$
\begin{gathered}
\Omega^{3}=0, \quad \Omega_{1}^{3}=\Omega^{1}, \quad \Omega_{2}^{3}=0, \quad \Omega_{2}^{1}=\Omega^{1}, \quad 3 \Omega_{1}^{1}+\Omega_{2}^{2}=\Omega^{2}, \\
\Omega_{1}^{1} \wedge \Omega^{1}=0, \quad\left(\Omega_{1}^{2}-\Omega_{3}^{1}\right) \wedge \Omega^{1}=\Omega_{1}^{1} \wedge \Omega^{2} .
\end{gathered}
$$

Let $i: L_{3} \rightarrow G$ be the inclusion mapping, and set $\omega^{i}=i^{*} \Omega^{i}, \omega_{j}^{i}=i^{*} \Omega_{j}^{i}$. Then $\left(\omega^{1}, \omega^{2}, \omega_{1}^{2}, \omega_{3}^{2}\right)$ is a coframe field on $L_{3}$, and

$$
\begin{aligned}
& \omega^{3}=0, \quad \omega_{1}^{3}=\omega^{1}, \quad \omega_{2}^{3}=0, \quad \omega_{2}^{1}=\omega^{1}, \quad \omega_{1}^{1}=-m \omega^{1}, \\
& \omega_{2}^{2}=3 m \omega^{1}+\omega^{2}, \quad \omega_{3}^{1}=\omega_{1}^{2}-p \omega^{1}-m \omega^{2},
\end{aligned}
$$

for some functions $m$ and $p$ on $L_{3}$; and $m$ is constant on the fibers of $L_{3}$. The function $m$, which is actually a function on $S$, is the fourth order invariant of $f$.

Lemma 8. Second order G-deformations of $f$ preserve the fourth order invariant $m$ of $f$. 
Proof. Suppose that $\bar{f}: S \rightarrow G$ is a second order $G$-deformation of $f$ with a deformation given by $v: S \rightarrow G$. That is, $v(s) \circ f$ agrees to second order with $\bar{f}$ at $s$, for each $s \in S$. Let $u: S \rightarrow L_{3}$ be a third order frame field along $f$, so that

$$
\begin{aligned}
& u^{*} \Omega^{3}=0, \quad u^{*} \Omega_{1}^{3}=u^{*} \Omega^{1}, \quad u^{*} \Omega_{2}^{3}=0, \\
& u^{*} \Omega_{2}^{1}=u^{*} \Omega^{1}, \quad u^{*} \Omega_{1}^{1}=-m u^{*} \Omega^{1} .
\end{aligned}
$$

Define $\bar{u}: S \rightarrow G$ by $\bar{u}(s)=v(s) u(s)$, so that $\bar{u}$ is at least a second order frame field along $\bar{f}$. But (19) and (42) show that equations (43) hold with bars on everything, so that $\bar{u}$ is actually a third order frame field along $\bar{f}$. But then using (19) we have

$$
\bar{u}^{*} \Omega_{1}^{1}=u^{*} \Omega_{1}^{1}=-m u^{*} \Omega^{1}=-m \bar{u}^{*} \Omega^{1}
$$

which implies that $m$ is the fourth order invariant of $\bar{f}$. q.e.d.

All Type IIb IIIb surfaces have the same fourth order type, but at the fifth order they split into two types, depending on whether $m=0$ (Type IIb IIIb Va), or $m \neq 0$ (Type IIb IIIb Vb). From Lemma 8 it follows that second order deformation preserves the fifth order type.

Proposition 10. (i) Any two Type IIb IIIb Va surfaces are second order $G$-deformations of each other.

(ii) Any two Type IIb IIIb Vb surfaces are second order G-deformations of each other whenever their fourth order invariants have the same range, i.e., whenever $m(S)=\bar{m}(\bar{S})$.

Proof. (i) Let $f: S \rightarrow \mathbf{R}^{3}$ be a Type IIb IIIb Va surface, and let $L_{3}$ be its bundle of third order frames. Using the notation preceding Lemma 8, we have (42) with $m=0$. Let $\bar{f}: S \rightarrow \mathbf{R}^{3}$ be another Type IIb IIIb Va surface with bundle of third order frames $\bar{L}_{3}$.

To get a second order deformation of $f$ to $\bar{f}$ it suffices, by (19), (42) and $(\overline{42})$, to find a two-dimensional solution of the closed exterior differential system on $L_{3} \times \bar{L}_{3}$ with specified independent variables $\omega^{1}, \omega^{2}$ :

$$
\bar{\omega}^{1}=\omega^{1}, \quad \bar{\omega}^{2}=\omega^{2}, \quad \bar{\omega}_{1}^{2}=\omega_{1}^{2}, \quad\left(\bar{\omega}_{3}^{2}-\omega_{3}^{2}\right) \wedge \omega^{1}=0 .
$$

This system is in involution, and the general solution depends on one function of one variable. The rest of the proof of (i) follows the lines of the proof of Proposition 9.

(ii) Let $f: S \rightarrow \mathbf{R}^{3}$ be a Type IIb IIIb Vb surface. Continue to use the notation of (42).

Lemma 9. There exists a unique third order frame field $u: S \rightarrow L_{3} \subseteq G$ along $f$ for which

$$
d m=-2 m \varphi^{2}, \quad \varphi_{1}^{2}=3 m \varphi^{2},
$$

where $\varphi^{i}=u^{*} \omega^{i}, \varphi_{j}^{i}=u^{*} \omega_{j}^{i}$. 
Proof. Let $u: S \rightarrow L_{3}$ be any third order frame field along $f$, and set $\varphi^{i}=u^{*} \omega^{i}, \varphi_{j}^{i}=u^{*} \omega_{j}^{i}$. Apply $u^{*}$ to (42) to get

$$
\begin{gathered}
\varphi^{3}=0, \quad \varphi_{1}^{3}=\varphi^{1}, \quad \varphi_{2}^{3}=0, \quad \varphi_{2}^{1}=\varphi^{1}, \\
\varphi_{1}^{1}=-m \varphi^{1}, \quad \varphi_{2}^{2}=3 m \varphi^{1}+\varphi^{2} .
\end{gathered}
$$

Then $0=d\left(\varphi_{1}^{1}+m \varphi^{1}\right)$ implies that

$$
d m \wedge \varphi^{1}=2 m \varphi^{1} \wedge \varphi^{2}
$$

from which we get

$$
d m=m_{1} \varphi^{1}-2 m \varphi^{2},
$$

where $m_{1}$ is some function on $S$, and depends on the choice of $u$.

Any other third order frame along $f$ is given by

$$
\tilde{u}=u \cdot\left[\begin{array}{lll}
1 & 0 & a \\
a & 1 & b \\
0 & 0 & 1
\end{array}\right],
$$

where $a$ and $b$ are arbitrary functions on $S$, (cf. [7, p. 129]). Let $\tilde{\varphi}^{i}=\tilde{u}^{*} \omega^{i}$, $\tilde{\varphi}_{j}^{i}=\tilde{u}^{*} \omega_{j}^{i}$. Then equations (46) hold for $\tilde{\varphi}^{i}$ and $\tilde{\varphi}_{j}^{i}$, and

$$
d m=\tilde{m}_{1} \tilde{\varphi}^{1}-2 m \tilde{\varphi}^{2} .
$$

Write $u=\left(e_{1}, e_{2}, e_{3} ; f\right)$ and $\tilde{u}=\left(\tilde{e}_{1}, \tilde{e}_{2}, \tilde{e}_{3} ; f\right)$, so that $\tilde{e}_{1}=e_{1}+a e_{2}, \tilde{e}_{2}=$ $e_{2}, \tilde{e}_{3}=a e_{1}+b e_{2}+e_{3}$. Then $d f=\varphi^{1} e_{1}+\varphi^{2} e_{2}=\tilde{\varphi}^{1} \tilde{e}_{1}+\tilde{\varphi}^{2} e_{2}$ implies that

$$
\tilde{\varphi}^{1}=\varphi^{1}, \quad \tilde{\varphi}^{2}=\varphi^{2}-a \varphi^{1} .
$$

Combining (47), (48) and (49) gives

$$
\tilde{m}_{1}=m_{1}-2 a m .
$$

Hence, by taking $a=\frac{1}{2} m_{1} / m$, the frame field $\tilde{u}$ will have the property that $d m=-2 m \tilde{\varphi}^{2}$.

We may assume then that $m_{1}=0$; i.e., that $d m=-2 m \varphi^{2}$. In order for the new frame field $\tilde{u}$ to share this property we must take $a=0$. Now $\tilde{e}_{1}=e_{1}$, $\tilde{e}_{2}=e_{2}, \tilde{e}_{3}=b e_{2}+e_{3}$, and

$$
\tilde{\varphi}^{1}=\varphi^{1}, \tilde{\varphi}^{2}=\varphi^{2}
$$

Differentiating $d m+2 m \varphi^{2}=0$ gives

$$
\varphi^{1} \wedge \varphi_{1}^{2}=3 m \varphi^{1} \wedge \varphi^{2} \text {. }
$$

Hence

$$
\varphi_{1}^{2}=k \varphi^{1}+3 m \varphi^{2}
$$

for some function $k$ on $S$.

As for the frame field $\tilde{u}$,

$$
d \tilde{e}_{1}=\tilde{\varphi}_{1}^{1} \tilde{e}_{1}+\tilde{\varphi}_{1}^{2} \tilde{e}_{2}+\tilde{\varphi}_{1}^{3} \tilde{e}_{3}=\tilde{\varphi}_{1}^{1} e_{1}+\left(\tilde{\varphi}_{1}^{2}+b \tilde{\varphi}_{1}^{3}\right) e_{2}+\tilde{\varphi}_{1}^{3} e_{3},
$$


and $d \tilde{e}_{1}=d e_{1}=\varphi_{1}^{1} e_{1}+\varphi_{1}^{2} e_{2}+\varphi_{1}^{3} e_{3}$. Hence

$$
\tilde{\varphi}_{1}^{2}=\varphi_{1}^{2}-b \tilde{\varphi}_{1}^{3}=\varphi_{1}^{2}-b \varphi^{1},
$$

because $\tilde{\varphi}_{1}^{3}=\tilde{\varphi}^{1}=\varphi^{1}$. Now $\tilde{\varphi}_{1}^{2}=\tilde{k} \varphi^{1}+3 m \varphi^{2}$, so that (50) and (51) imply

$$
\tilde{k}=k-b \text {. }
$$

By taking $b=k$ we can make $\tilde{k}=0$. This proves Lemma 9, and permits us to complete the proof of Part (ii) of Proposition 10.

Let $\bar{f}: \bar{S} \rightarrow \mathbf{R}^{3}$ be any Type IIb IIIb Vb surface, and let $u: S \rightarrow L_{3}, \bar{u}$ : $\bar{S} \rightarrow \bar{L}_{3}$ be the special third order frame fields along $f$ and $\bar{f}$, respectively, given by Lemma 9 . Set $\bar{\varphi}^{i}=\bar{u}^{*} \bar{\omega}^{i}$ and $\bar{\varphi}_{j}^{i}=\bar{u}^{*} \bar{\omega}_{j}^{i}$. Then (45) and (46) hold for both $u$ and $\bar{u}$, where we let $\bar{m}$ denote the fourth order invariant of $\bar{f}$.

The exterior differential system

$$
\bar{\varphi}^{1}=\varphi^{1}, \bar{\varphi}^{2}=\varphi^{2}
$$

on $S \times \bar{S}$ is completely integrable. Choose points $o \in S$ and $\bar{o} \in \bar{S}$ such that $m(o)=\bar{m}(\bar{o}) \neq 0$, and let the graph of $F: S \rightarrow \bar{S}$ be the two-dimensional integral manifold of (53) for which $F(o)=\bar{o}$. Then

$$
F^{*} \bar{\varphi}^{1}=\varphi^{1}, \quad F^{*} \bar{\varphi}^{2}=\varphi^{2},
$$

and

$$
F^{*} d \log |\bar{m}|=F^{*}\left(-2 \bar{\varphi}^{2}\right)=-2 \varphi^{2}=d \log |m|
$$

implies that

$$
\bar{m} \circ F=c \cdot m, \quad \text { for some constant } c .
$$

But $c=1$, because $\bar{m} \circ F(o)=\bar{m}(\bar{o})=m(0) \neq 0$.

Combining (45), (46), (54) and (55) it follows that equations (29) hold for the frame fields $u$ and $\bar{u}$. Hence, by Part(b) of Proposition $6, \bar{f}$ is a second order $G$-deformation of $f$.

Remark. Type IIb IIIb Va surfaces are cones, while Type IIb IIIb Vb surfaces are tangent developables. To see this, let $u=\left(e_{1}, e_{2}, e_{3} ; f\right)$ be a third order frame field on the surface with relative components of infinitesimal displacement $\varphi^{i}=u^{*} \omega^{i}, \varphi_{j}^{i}=u^{*} \omega_{j}^{i}$, where $\omega^{i}, \omega_{j}^{i}$ are the forms on $L_{3}$ in (42).

For a Type IIb IIIb Va surface the invariant $m$ is identically zero, so that equations (42) imply that

$$
\begin{aligned}
d f & =\varphi^{1} e_{1}+\varphi^{2} e_{2}, \\
d e_{2} & =\omega_{2}^{1} e_{1}+\omega_{2}^{2} e_{2}+\omega_{2}^{3} e_{3}=\varphi^{1} e_{1}+\varphi^{2} e_{2} .
\end{aligned}
$$

Hence $d\left(f-e_{2}\right)=0$, and so $f=c+e_{2}$ which is a cone with center at $c \in \mathbf{R}^{3}$ and generator lines parallel to $e_{2}$. 
For a type IIb IIIb Vb surface the invariant $m$ is nonconstant, and equations (42) give

$$
d e_{2}=\varphi^{1} e_{1}+\left(3 m \varphi^{1}+\varphi^{2}\right) e_{2}=d f+3 m \varphi^{1} e_{2} .
$$

The vector field $e_{2}$ is the tangent vector along any curve $\varphi^{1}=0$, and it does not change direction along such a curve; these curves are straight lines, the generator lines of the surface. Along any of the lines $\varphi^{1}=0$ we have

$$
d e_{1}=3 m \varphi^{2} e_{2}
$$

from which it follows that the tangent plane to the surface is constant along a generator line. Hence $f$ is a developable. It is easily seen that any cylinder is of Type IIb IIIa, while any cone is Type IIb IIIb Va. Thus any Type IIb IIIb Vb surface must be a tangent developable, (or osculating developable, as it is called sometimes).

\section{References}

[1] E. Cartan, Sur la déformation projective des surfaces, Ann. Sci. École Norm. Sup. 37 (1920) 259-356; or Oeuvres Complètes, III 1, 441-538.

[2] __ Sur le problème général de la déformation, C. R. Congrès Strasbourg, 1920, 397-406.

[3] _ Théorie des groupe finis et continues et la géométrie différentielle traitées par la méthode du repère mobile, Gauthier-Villars, Paris, 1937.

[4] __ Les systèmes différentiels extérieurs et leurs applications géométriques, Hermann, Paris, 1945.

[5] K. F. Gauss, General investigations of curved surfaces, Raven Press, Hewlett, New York, 1965.

[6] P. Griffiths, On Cartan's method of Lie groups and moving frames as applied to uniqueness and existence questions in differential geometry, Duke Math. J. 41 (1974) 775-814.

[7] G. R. Jensen, Higher order contact of submanifolds of homogeneous spaces, Lecture Notes in Math. Vol. 610, Springer, Berlin, 1977.

[8] S. Kobayashi \& K. Nomizu, Foundations of differential geometry, Vol. I, Interscience, New York, 1963.

[9] C. Schiemangk \& R. Sulanke, Submanifolds of the Möbius space, Math. Nach., to appear.

[10] R. Sulanke, On E. Cartan's method of moving frames, to appear in Proc. Colloq. Differential Geometry, Budapest, 1979.

[11] R. Sulanke \& A. Svec, Zur differentialgeometrie der untermannigfaltigkeiten eines Kleinschen Raumes, Beitrage zur Algebra und Geometrie, Halle, to appear.

[12] H. Weyl, Cartan on groups and differential geometry, Bull. Amer. Math. Soc. 44 (1938) 598-601. 